\title{
Zones marginales des études postcoloniales : nouvelles approches et comparaisons entre les mondes indien et russo-soviétique
}

Philippe Bornet et Svetlana Gorshenina

\section{CpenEdition}

Journals

Édition électronique

URL : http://journals.openedition.org/edl/645

DOI : $10.4000 /$ edl. 645

ISSN : 2296-5084

Éditeur

Université de Lausanne

\section{Édition imprimée}

Date de publication : 15 septembre 2014

Pagination : 17-78

ISBN : 978-2-940331-35-2

ISSN : 0014-2026

Référence électronique

Philippe Bornet et Svetlana Gorshenina, «Zones marginales des études postcoloniales : nouvelles approches et comparaisons entre les mondes indien et russo-soviétique », Études de lettres [En ligne], 2-3 | 2014, mis en ligne le 15 septembre 2017, consulté le 18 décembre 2020. URL : http:// journals.openedition.org/edl/645; DOI : https://doi.org/10.4000/edl.645 


\section{ZONES MARGINALES DES ÉTUDES POSTCOLONIALES : NOUVELLES APPROCHES ET COMPARAISONS ENTRE LES MONDES INDIEN ET RUSSO-SOVIÉTIQUE}

\section{De la donne institutionnelle au défi méthodologique}

Alors que son idée remonte à plusieurs années, le colloque qui a donné lieu à la présente publication a été profilé pour marquer la réunion, au sein de la Faculté des lettres de l'Université de Lausanne, des sections des langues et civilisations slaves et des langues et civilisations orientales ${ }^{1}$. Cette mutualisation a eu pour résultat la création d'une nouvelle section de langues et civilisations slaves et de l'Asie du Sud. Forte de deux pôles de compétences, la Russie et l'Inde, cette section couvre finalement

I. Au départ en 2011, le projet ne portant que sur l'orientalisme russo-soviétique a bénéficié de réflexions communes menées avec Sergej Abašin, Vladimir Bobrovnikov, David Schimmelpenninck van der Oye et Vera Tolz. Par la suite, les enjeux intellectuels du projet ont été ajustés dans une perspective comparative russo-indienne, notamment à la faveur de discussions avec Maya Burger et Patrick Sériot. Nous adressons toute notre reconnaissance à ces collègues pour ce partage généreux de leurs idées et expériences. Nous remercions également Claude Rapin et Vera Tolz pour leurs précieuses remarques sur une version préliminaire de ce texte. En outre, nous aimerions remercier également les institutions qui ont soutenu le colloque et la présente publication, à savoir la Faculté des lettres et le Département Interfacultaire d'Histoire et de Sciences des Religions de l'Université de Lausanne, le Fonds national suisse de la recherche scientifique et la revue Etudes de Lettres. Nos vifs remerciements enfin aux six recenseurs anonymes dont les réflexions ont largement contribué à la qualité du volume. Si le présent ouvrage ne reproduit pas la totalité des contributions présentées au colloque des 14-17 mai 2013 à l'Université de Lausanne notamment celles de Vladimir Bobrovnikov, Anke von Kügelgen, Sébastien Moret, Patrick Sériot et Aleksej Vigasin, il est possible de retrouver certaines de ces communications en ligne: $<$ https://itunes. apple.com/ch/itunes-u/lorientalisme-des-marges-colloque/id676060638?l=fr\&mt=10>. 
une aire aux contours inédits qui s'étale, à travers l'Eurasie, de l'Europe orientale au Sri Lanka et au Bangladesh.

Cette structure possède cependant, dans l'histoire de l'Université de Lausanne, un illustre précurseur personnifié dans la figure de l'un de ses anciens professeurs ordinaires, Constantin Regamey (1907-1982) ${ }^{2}$. Musicien, russisant et indologue à la fois, surdoué et d'un profil académique fortement atypique, il a été à la source de l'enseignement des langues slaves et indiennes à Lausanne. Né à Kiev, il a été formé en Pologne auprès de Stanislas Schayer (1899-1941) et à Paris, notamment auprès de Sylvain Lévi (1863-1935). Il quitte la Pologne en 1944 pour s'installer à Lausanne. Tout en développant une riche activité de compositeur, il occupera dès 1945 une chaire de "civilisations slaves et orientales" créée expressément à son attention en Faculté des lettres. L'intitulé de cette chaire tout comme ses nombreux travaux portant sur des sujets extrêmement variés témoignent de l'activité d'un grand érudit et humaniste ${ }^{3}$, marquée par la rigueur propre à un spécialiste de nombreuses langues - parmi lesquelles le tibétain, le sanscrit et le russe.

En dépit des travaux de ce pionnier et de la volonté souvent affichée d'encourager les études transversales en faveur de l'inter- et de la pluridisciplinarité, la détermination d'une thématique susceptible d'intéresser l'ensemble des membres de la nouvelle section n'a pas été immédiatement évidente. Le prétexte formel découlant de la restructuration administrative survenue au sein de l'Université de Lausanne s'est ainsi transformé en un défi méthodologique, conduisant à encourager la fécondité des regards réciproques et définir un sujet commun.

Le questionnement sur l'orientalisme, pris ici dans le sens ébauché par Edward Saïd (1935-2003) ${ }^{4}$ constitue une plateforme fédératrice qui, à l'interface de plusieurs problématiques régionales et disciplinaires,

2. Pour une présentation sommaire des activités de Regamey et une bibliographie de ses travaux, voir C. Regamey, "Hommage à Constantin Regamey».

3. Voir, p. ex., ses articles comparatifs entre pensée indienne et pensée européenne: C. Regamey, "The Meaning and Significance of Spirituality in Europe and in India".

4. E. Said, Orientalism et Culture and Imperialism. Même si la notion même d'orientalisme n'est pas univoque - y compris chez Saïd lui-même et chez ses critiques comme Aijaz Ahmad (1932-), John M. MacKenzie (1943-) ou Robert Irwin (1946-) - il est indéniable que le terme a fédéré depuis Saïd un ensemble de débats concernant le statut des études savantes sur les Asie(s) et les représentations (y compris populaires) de l'«Orient» en Europe. 
permet de construire de nouvelles approches tout en désenclavant les études portant sur ces aires. Le choix de cette problématique était d'autant plus logique que la section dispense depuis 2011 un enseignement sur l'orientalisme académique en rapport avec l'Inde.

En même temps, la compréhension de l'orientalisme telle qu'envisagée dans le présent ouvrage ne se limite pas uniquement aux études savantes et à leurs corrélations avec les agendas politiques, mais englobe également les techniques politiques de soumission de l'Autre, les réminiscences littéraires ou artistiques des expériences coloniales, la vulgarisation des théories scientifiques et les visions populaires qui en résultent.

En outre, il s'avère que, dans les traditions académiques actuelles, les "passerelles» entre les mondes slaves et indiens d'une manière générale (et non uniquement sur la thématique de l'orientalisme) sont loin d'être nombreuses et les exemples existants sont plutôt marginaux.

\section{Regards réciproques Russie-Inde: une histoire à plusieurs vitesses}

\subsection{L'intérêt pour l'Inde en Russie et l'indologie soviétique}

Pressenti durant les siècles précédents, l'intérêt stratégique pour l'Inde en Russie ne s'est manifesté de manière claire et soutenue que dans les premières ébauches géopolitiques de l'époque de Pierre le Grand (16721725).

Toutefois, les études académiques sur cette région, ciblées initialement, comme en Europe, sur le sanscrit et ensuite, de manière plus originale, sur le bouddhisme ${ }^{5}$, n'ont démarré véritablement que vers la fin du premier tiers du XIX ${ }^{e}$ siècle, donc avec un certain retard par rapport aux autres branches des disciplines académiques s'intéressant à l'Orient (vostokovedenie) $^{6}$. Soumis pendant pratiquement toute la période tsariste

5. A cette époque, les études indiennes en Europe portent plutôt leur attention sur l'Inde védique. La précocité de l'intérêt pour le bouddhisme en Russie s'explique par la présence sur le territoire impérial des Bouriates-Mongols, eux-mêmes très tôt associés aux études de cette religion par les chercheurs de Saint-Pétersbourg, notamment F. I. Ščerbatskij.

6. Hébergé initialement parmi les disciplines de l'Académie des sciences (Robert Lenz [1808-1836]), le sanscrit commence à être enseigné dans le cadre universitaire d'abord à Kazan en 1841 (Pavel Ja. Petrov [d. 1876]), puis à Moscou (le même Petrov 
à une forte influence des savants allemands, français et britanniques, l'indologie russe n'est pas parvenue à s'émanciper de sa position marginale du fait qu'elle s'est développée dans une situation où l'intérêt de l'empire - et de ses chercheurs - s'est porté principalement sur l'Asie centrale et la Chine.

Reflétant cette situation au niveau du public, les intellectuels russes - partie prenante des discussions entre slavophiles et occidentalistes - ne s'intéressent pas à l'histoire indienne (qui leur est d'ailleurs pratiquement inconnue) lorsqu'ils réfléchissent sur l'identité nationale russe. De plus, la perception de l'Inde en Russie reste marquée par une vision dénigrante héritée de l'orientalisme britannique du début du XIX ${ }^{\mathrm{e}}$ siècle ${ }^{7}$, comme on l'observe même chez les écrivains et hommes politiques "progressistes» Vissarion G. Belinski (1811-1848), Pëtr Ja. Tchaadaïev (17941856) ou Alexandre I. Herzen (1812-1870).

Ultérieurement, l'indologie académique russo-soviétique s'occupe tout d'abord et plutôt à Saint-Pétersbourg de la traduction érudite des textes anciens, dont une partie provient des fouilles archéologiques au Turkestan chinois ; l'école saint-pétersbourgeoise de Fëdor I. Ščerbatskij (1866-1942) est pendant des décennies l'une des plus célèbres du monde, surtout dans le domaine des études bouddhistes ${ }^{8}$. Durant la première moitié du XX $\mathrm{XX}^{\mathrm{e}}$ siècle, cette discipline se déplace partiellement à Moscou où elle se concentre essentiellement sur la recherche interprétative des conditions sociales, politiques ou économiques du sous-continent

entre 1852-1875) et, en 1856, à Saint-Pétersbourg, où une spécialisation "sanscritotibétaine» est officiellement créée en tant que sixième branche des études orientales de la Faculté orientale de l'Université: V. V. Bartol'd, Istorija izučenija Vostoka v Evrope iv Rossii, p. 463 sq.; G. N. Roerich, «Indology in Russia»; A. P. Bazijanc, D. E. Bertel's, Aziatskij muzej, p. 151-154; G. M. Bongard-Levin, A. A. Vigasin, The Image of India, p. 95 sq., 103-107; E. I. Zelenev, V. B. Kasevič, Vvedenie v vostokovedenie, p. 13. De manière générale, il convient de souligner l'absence d'une histoire détaillée et non idéologisée des études indiennes en Russie et en Union soviétique.

7. Voir à ce propos Th. R. Trautmann, Aryans and British India, p. 99-130 («British Indophobia»).

8. C'est à Saint-Pétersbourg que sera publié le célèbre dictionnaire d'Otto von Böhtlingk et Rudolph von Roth, Sanskrit Wörterbuch, entre 1855 et 1875 . On peut également mentionner la collection Bibliotheca Buddhica qui est lancée dans la même ville en 1897 par Sergej F. Ol'denburg (1863-1934) et F. I. Ščerbatskij. 
indien ${ }^{9}$. Mortifiée à plusieurs reprises par le pouvoir soviétique ${ }^{10}$, cette discipline, où connaissances solides et clichés politiques se mêlent alors constamment, n'a pas pu s'émanciper et les comparaisons entre la Russie et l'Inde sont demeurées rares, et généralement empreintes de présupposés idéologiques majeurs.

Pendant ces deux siècles, l'importance des regards réciproques par rapport à la Russie et à l'Inde est sensiblement inégale. Les tentatives de comparaison apparaissent tout d'abord dans le domaine de la linguistique avec des analyses des parallèles entre le russe et le sanscrit, deux langues inscrites dans la large famille des langues indo-européennes conformément au mainstream de la linguistique occidentale du XIX ${ }^{e}$ siècle. Associée aux précurseurs des indologues russes Gerasim S. Lebedev (1749-1817) et Friedrich P. von Adelung (1768-1843), la première vague du comparatisme russo-indien, "aryenne", débouche rapidement - non seulement en Russie, mais également au niveau international - sur des essais politistes spéculant sur les avancées impérialistes russes et britanniques au Turkestan et en Inde et sur une rencontre éventuelle des deux puissances sur le "Toit du monde» ${ }^{11}$.

Indépendamment des projets coloniaux, la fondation en 1875 de la Société théosophique aux Etats-Unis (la branche russe ne s'ouvre qu'en 1908) et la publication en 1888 de La doctrine secrète d'Helena P. Blavatsky [Blavatskaja] (1831-1891) sont à la racine d'une nouvelle fascination pour l'Inde en Russie. Quelques cercles d'intellectuels (et surtout d'intellectuelles parmi lesquelles figure l'influente Helena Roerich

9. D. A. Birman, G. G. Kotovskij, N. N. Sosina, Bibliografija Indii.

Io. Plusieurs vagues de répressions staliniennes ayant touché l'ensemble des chercheurs spécialisés dans les études orientales; plus précisément par rapport à l'indologie, le procès - assez tardif, mais spectaculaire - du spécialiste bouriate du bouddhisme Badija D. Dandaron (1914-1974), amène à considérer que les études du bouddhisme et par extension de toutes les religions nées en Inde sont potentiellement dangereuses en Union soviétique: A. A. Vigasin, "Istoriografija istorii drevnej Indii», p. 107; Ja. V. Vasil'kov, M. Ju. Sorokina, Ljudi i sud'by; V. Lysenko, "Oktjabrina Fëdorovna Volkova», p. 12.

II. Pour un bref historique de ces réflexions géopolitiques rédigées dans l'esprit du Great Game à travers la lutte des deux puissances pour la soumission du cœur de l'Asie voir A. S. Morrison, Russian Rule in Samarkand, p. 5-7. Sur le Great Game et sur les spéculations relatives aux "frontières naturelles" des deux puissances voir, de manière plus détaillée, S. Gorshenina, Asie centrale, p. 37-93, 145-172 (pour les références bibliographiques, voir notamment la note 179). 
[1879-1955], épouse de Nicolas Roerich [1874-1947] et l'une des fondatrices de l'Agni Yoga Society) se mettent à la recherche du savoir ésotérique supposé né en Inde en des temps immémoriaux et diversement préservé en Occident et en Orient. A travers le bouddhisme essentiellement, cette recherche appelle à une large synthèse religieuse entre "Occident" et "Orient", conçue par référence (explicite ou non) à la tradition orientaliste européenne ${ }^{12}$. La comparaison s'élargit donc au-delà de l'Inde et de la Russie, mais le but revient finalement à revivifier la spiritualité en Russie par la source indienne.

Considérés comme "bourgeois", ces sujets ont à l'époque soviétique cédé la place à des études sur les explorations menées en Inde par les voyageurs russes, à commencer par le très célèbre Afanasij Nikitin (14661472). A l'exemple des premières compilations publiées dans les années 1940-1950 par Nikolaj M. Gol'dberg (1891-1961) et Koka A. Antonova (1910-2007), ces recueils - afin de se protéger des reproches politiques? - sont restés en grande partie limités à l'édition de documents d'archives ${ }^{13}$, même si le diapason chronologique s'est étalé de l'Antiquité au début du $\mathrm{XX}^{\mathrm{e}}$ siècle avec des publications allant de thèmes spécialisés à des thèses sur l'historique de ce type d'études ${ }^{14}$.

Hébergée de temps à autre dans le cadre des études "culturologiques", la problématique des relations russo-indiennes devient à la mode dans les années 1990 quand elle est associée aux analyses des représentations

I2. M. Carlson, "No Religion Higher than Truth"; M. Laruelle, Mythe aryen et rêve impérial dans la Russie tsariste, p. 125-131. On peut notamment évoquer les activités coordonnées à Saint-Pétersbourg par Anna Kamenskaya (1867-1952) et Elena F. Pisareva (1855-1944). Dès après la révolution russe, les travaux de la Société russe de théosophie sont considérés comme incompatibles avec le rationalisme soviétique et la Société doit s'exiler jusque dans les années 1990. Anna Kamenskaya parvient à s'enfuir avant d'être arrêtée et s'établit en Suisse où lui sera confiée une charge de cours à l'Université de Genève à partir de 1926. Traductrice de la Bhagavadgìtā en russe et en français, elle enseignera sur différents sujets en histoire comparée des religions.

I3. Dans l'importante série de publications de ce type voir, p. ex., la première et les dernières parutions: K. A. Antonova, N. M. Gol'dberg, T. D. Lavrencova, Russkoindijskie otnošenija v XVII v. Sbornik dokumentov; A. A. Vigasin et al., Russko-indijskie otnošenija v XIX v. Sbornik arhivnyh dokumentov i materialov; A. A. Vigasin et al., Russko-indijskie otnošenija v 1900-1917 gg. Sbornik arhivnyh dokumentov i materialov; E. L. Belov, O. I. Svjateckaja, T. L. Šaumjan, Rossija i Tibet.

I4. G. F. Mrathuzina, Rossijsko-indijskie otnošenija v otečestvennoj istoriografii. Pour une étude récente (2013) de ces figures de voyageurs russes en Inde, voir A. Mukhopadhyay, India in Russian Orientalism. 
indiennes - fortement exotisées et romantisées - dans la littérature et les arts russes. Dans cette optique, les rapports entre la Russie et l'Inde se placent dans la lignée des idées que Mikhaïl I. Venjukov (1832-1901) et Andrej E. Snesarev (1865-1937), notamment, formulent au XIX ${ }^{\mathrm{e}}$ siècle à travers une critique farouche du colonialisme britannique qu'ils opposent à l'attitude russe; conçus comme un "dialogue», une "interaction", ces rapports sont considérés comme un ensemble de «liens» fondés sur une sympathie réciproque et une "parenté originelle», pratiquement "innée", des deux pays ${ }^{15}$. Souvent entreprises hors de toute contextualisation historique, ces études ont été fortement influencées par les travaux portant sur l'«impérialisme britannique» qui, à côté des recherches relatives aux systèmes économiques en Inde, occupent le sommet du palmarès de l'indologie soviétique ${ }^{16}$. Sans surprise et conformément aux intérêts «internationalistes» de l'Union soviétique, les échanges épistolaires entre Lev N. Tolstoï (1828-1910) et Mohandas K. Gandhi (1869-1948) autour du concept de désobéissance civile et non violente ont servi à mettre en lumière le rôle "progressiste» de la Russie face au "sous-développement" indien.

On peut ajouter à cela un intérêt pour certains aspects de la littérature produite en Inde comme, par exemple, plusieurs textes de Rabindranath Tagore (1861-1941). Ce dernier connaît une influence grandissante dans la Russie prérévolutionnaire grâce à la traduction russe en 1914 de son recueil de poèmes Gìtānjali, dont l'original bengali paraît en 1910 et la version anglaise qui lui vaut le prix Nobel de littérature en 1913. Le gouvernement soviétique contribue par la suite à la diffusion des œuvres de Tagore, qu'il présente comme un fervent défenseur du régime et compare souvent à Tolstoï. Dès les années 1950, des traductions russes d'œuvres hindies comme celles de Premchand (1880-1936), Yashpal (1903-1976) ou Nirmal Verma (1929-2005) circulent couramment ${ }^{17}$.

Bien que le poids des anciennes approches idéologisées, notamment l'idée de la "grande amitié des peuples» qui a constamment marqué les relations russo-indiennes, ainsi qu'un positivisme souvent primaire

I5. Voir, p. ex., M. A. Korjukaeva, Russko-indijskie kul'turnye svjazi vtoroj poloviny $X I X-$ načala $X X v v$.

I6. Comme exemple, voir N. A. Halfin, P. M. Šastitko, Indija i Rossija; G. G. Kotovskij, A. N. Hejfetc, P. M. Šastitko, SSSR i Indija.

17. Voir à ce propos G. Rousseva-Sokolova, "Behind and Beyond the Iron Curtain", p. 236-239. 
se fassent toujours sentir, quelques publications récentes proposent un regard transversal et une perspective comparatiste sur des sujets comme les recherches spirituelles entreprises par diverses personnalités russes marquantes (comme H. P. Blavatsky, L. N. Tolstoï, N. Roerich), des interprétations nouvelles des motifs indiens dans la littérature russe, des épisodes du Great Game ou les mouvements migratoires réciproques entre les deux pays ${ }^{18}$.

2.2. L'intérêt pour la Russie en Inde et les slavistes indiens: à la recherche d'un modèle

La trajectoire en Inde de l'intérêt à l'égard de la Russie est, compte tenu de la situation géopolitique, différente. Lancé vers la fin des années 1810 par quelques intellectuels indiens intéressés par la figure de Pierre le Grand, ce mouvement s'est, comme partout dans le monde, davantage concentré vers le début du $\mathrm{XX}^{\mathrm{e}}$ siècle sur des études de la littérature russe essentiellement représentée par les grands écrivains du siècle précédent L. N. Tolstoï (1828-1910), Anton P. Chekhov (1860-1904), Fëdor F. Dostoïevski (1821-1881) et Fëdor I. Tioutchev (1803-1873).

Dépendants au début de la situation coloniale, les savants indiens évoquent avant tout le cas russe en le mettant dans le contexte des relations indo-britanniques. Avant l'indépendance, la Russie fait figure de modèle socio-politique positif, par opposition à l'Angleterre. L'intérêt pour la Russie relève donc non seulement d'une critique de l'impérialisme britannique, mais plus largement de la recherche d'un «modèle de civilisation" qui puisse être opposé à un "Occident» essentialisé. C'est dans ces circonstances que se développe l'intérêt pour l'expérience politique soviétique. En 1920, le Parti communiste indien se constitue progressivement à la faveur d'un congrès que ses futurs membres réunissent en exil à Tachkent. Ce n'est cependant qu'en 1925, à la conférence de Kanpur, que le parti est officiellement fondé. Plusieurs de ses

I8. P. ex., E. Ju. Petrova, Rossijsko-indijskii istoričeskie svjazi v dokumentah i materialah arhivov L. N. Tolstogo i muzeja-usad'by "Jasnaja Poljana»; A. Andreev, Hram Buddy $v$ Severnoj stolice; S. Serebriany, «Le roman en Russie et en Inde»; D. Savelli, Présence du bouddhisme en Russie et "Des théosophes sur la route de Lhassa»; A. Stasulane, Theosophy and Culture; E. V. Fiskovec, Obraz Indii v russkoj literature. 
représentants séjourneront en Russie, comme Manabendra Nath Roy (1887-1954) qui sera reçu par Lénine et contribuera activement à la propagation de l'idéal communiste en dehors de la Russie dans le cadre du Komintern (Mexique, Chine, Inde) ${ }^{19}$. Pour des raisons évidentes, les activités du Parti communiste indien demeurent clandestines dans sa patrie jusqu'en 1942 et ses partisans seront régulièrement inquiétés par l'administration britannique.

La perception fortement idéalisée du système soviétique par Roy (qui révisera d'ailleurs ses positions quelques années plus tard en faveur d'un "humanisme radical») se retrouve sous une forme plus modérée chez R. Tagore. Invité à Moscou en 1930, il publie peu après des Letters from Russia qui sont censurées par l'administration britannique et publiées sous une forme expurgée en russe en $1956^{20}$. Tagore y dit son admiration du système éducatif russe, qu'il loue par contraste avec l'inaction de l'administration britannique face au problème de l'illettrisme en Inde. Il ne se gêne cependant pas d'émettre une critique à l'égard du système soviétique, dont il perçoit le risque pour les libertés individuelles.

Parmi les intellectuels indiens qui s'inspirent de l'exemple soviétique figure aussi Jawaharlal Nehru (1889-1964) qui s'intéresse à la Russie dès les années 1920, alors même que son envergure politique s'affirme au sein du parti du Congrès. Ainsi, dans son ouvrage Soviet Russia: Some Random Sketches and Impressions paru en 1928, il compare explicitement les relations entre l'Inde, la Russie et l'Angleterre ${ }^{21}$. Le rapport négatif à l'Angleterre n'influence toutefois pas l'image de l'empire russe que l'Inde voit à la fois comme un rival historique de l'Empire britannique et comme un pays de nature comparable à l'Inde, avec des économies similaires également centrées autour des paysans et de l'agriculture.

Après 1947, l'Inde post-indépendance est formellement associée au «bloc communiste» et fortement influencée à plusieurs niveaux - jusqu’à l'instauration de plans quinquennaux - par l'exemple soviétique. Le

19. Sur le parcours de Roy, voir la monographie de J. P. Haithcox, Communism and Nationalism in India, notamment p. 20-36, pour le congrès de Tachkent; également l'article de N. Pozza dans ce volume.

20. R. Tagore, Letters from Russia.

2I. J. Nehru, Soviet Russia, p. 131: "The continued friction that we see today is between England and Russia, not between India and Russia. Is there any reason why we in India should inherit the age long rivalry of England against Russia?» 
monde russe exerce aussi un attrait important et durable sur plusieurs auteurs hindis indiens, qui feront fréquemment le voyage de la Russie, tandis que l'association Indo-Soviet Cultural Society (ISCUS) se montre très active dans l'établissement des relations culturelles entre les deux pays $^{22}$.

Au niveau académique indien, le russe - s'il est enseigné - en reste bien souvent à sa dimension purement linguistique et est alors hébergé en département de "langues étrangères». En 1946 cependant, un Département de russe est inauguré à l'University of Delhi, précisément à l'initiative de $\mathrm{Nehru}^{23}$. Dès le départ, un objectif comparatif lui est assigné: les chercheurs indiens approchent alors l'histoire et la culture russes sous l'angle de la recherche de modèles socio-politiques adaptables au sous-continent et, notamment, cherchent à trouver une solution au problème des nationalités. Leur demande ne reste pas sans réponse: les politiciens et les chercheurs de l'URSS proposent alors - mais avec un succès mitigé - un certain nombre de scénarios de développement pour les pays non communistes fraîchement décolonisés ${ }^{24}$. Les études slaves se sont ainsi développées sur fond d'un projet politique clair et en opposition au modèle capitaliste et colonial incarné par l'Angleterre.

Créé sur cette même base au sein de la Jawahrlal Nehru University dans les années 1960, un Centre de russe et d'études centrasiatiques va logiquement s'intéresser aux questions de politique internationale et de relations bilatérales entre l'Inde et la Russie. Ce centre développe aujourd'hui ses activités sur les problématiques liées à la politique étrangère des anciennes républiques soviétiques, afin de faire ressortir leur point de vue, souvent considéré comme extrêmement marginal sur l'échiquier géopolitique de l'Asie ${ }^{25}$.

Bien que dans les travaux indiens sur la Russie, l'aspect politique soit préféré aux dimensions littéraires ou purement linguistiques, la

22. Pour les détails, voir P. M. Kemp, Bharat-Rus.

23. Pour l'historique du département, récemment renommé Department of Slavonic \& Finno-Ugrian Studies, voir le site <http:/www.du.ac.in/du/index. php?page=slavonic-finno-ugrian-studies $>$ (consulté le 24.06.2014).

24. S. Clarkson, «Le modèle soviétique de développement pour l'Inde» et «NonImpact of Soviet Writing on Indian Thinking and Policy».

25. Voir la page de présentation de ce centre qui a été récemment subdivisé en deux structures: Centre of Russian Studies (<http://www.jnu.ac.in/SLLCS/CRS/>) et Centre of Persian and Central Asian Studies (page en construction) (consulté le 24.06.2014). 
comparaison entre les littératures russe et indienne ne disparait pas pour autant de l'horizon des slavistes indiens ${ }^{26}$.

A l'issue de ce bref tour d'horizon des traditions des études réciproques dans les deux pays, force est de constater que la comparaison entre les mondes russe et indien, et en particulier sous l'angle des aspects induits par une situation coloniale ${ }^{27}$, est demeurée essentiellement implicite dans les travaux des universitaires russes et indiens. Ce constat est particulièrement saillant pour la problématique relative à la production des savoirs politiquement engagés, alors même qu'il existe plusieurs importantes thématiques transversales à exploiter.

\section{Les orientalismes en Russie et en Inde: une mise en parallèle d' "incomparables"}

\subsection{Lorientalisme en Russie: je t’aime, moi non plus}

L'intérêt pour l'orientalisme russe est initié par des historiens-russisants au moment où la Russie suscite un regain d'intérêt, une fois que celle-ci est reconnue sous sa forme d'empire multiethnique et multiconfessionnel.

Ces études débutent cependant par les doutes que Nathaniel Knight exprime en 2000 sur la possibilité même d'appliquer les méthodes forgées dans le sillage de Saïd à l'histoire de l'empire tsariste ${ }^{28}$. A la fois

26. Voir, p. ex., les séminaires régulièrement tenus à l'University of Delhi où parmi les sujets souvent explorés figure la comparaison entre les contes magiques russes et indiens (P. Das) et la poésie classique russe, notamment celle de F. Tioutchev avec la poésie marathie, de Tagore, de Nirala (M. Mittal, In the Soul's Twilight; M. Bade, D. Raghuvanshi, A. Bandyopadhyay, D. Dasgupta, Pande, A. Tripathi), des œuvres de Tolstoï avec celles de Tagore (T. Bhattacharya) ou celles d'A. Chekhov avec Premchand. Par symétrie, quoiqu'ils ne traitent pas souvent cette problématique transversale, les chercheurs occidentaux ont laissé quelques travaux sur les genres littéraires ou sur les motifs indiens chez des auteurs russes classiques (S. Sundaram, The Land of Thought, sur K. Bal'mont, ou R. H. Stacy, India in Russian Literature, qui s'apparente plutôt à un catalogue raisonné où l'Inde et l'"Orient en général" se chevauchent souvent; également A. Di Ruocco, The Buddhist World in Modern Russian Culture (1873-1919), sur les représentations du bouddhisme dans la littérature et les arts visuels russes).

27. Sur la notion de «situation coloniale», cf. G. Balandier, «La situation coloniale».

28. Pour l'historique de ces premières discussions et des références bibliographiques voir S. Gorshenina, "La marginalité du Turkestan colonial russe est-elle une fatalité, ou l'Asie centrale postsoviétique entrera-t-elle dans le champ des Post-Studies?", 
"objet» d'une science occidentale, qui la "ré-orientalise» en soulignant ses caractéristiques non européennes ${ }^{29}$, et productrice (donc "sujet») d'un discours "orientaliste" à l'égard de ses propres espaces périphériques méridionaux et à l'encontre d'un «Autre oriental " plus éloigné, la Russie commence par susciter un discours qui se concentre pour l'essentiel sur sa nature "euro-asiatique» fondée sur la continuité continentale de l'empire. Incompatible avec le modèle saïdien, cette dernière notion désoriente la première vague des interlocuteurs. Se faisant volontairement ou non écho du néoeurasianisme ${ }^{30}$, les discussions s'égarent dans une impasse provoquée par les croyances essentialistes en l'existence d'un «particularisme» non colonial russe et d'une expérience historique originale et sans précédent qui, en se renforçant, se serait propagée au monde soviétique ${ }^{31}$.

La traduction de l'Orientalisme de Saïd vers le russe prend place à une date particulièrement tardive ${ }^{32}$ et les approches des Post-Studies connaissent une forte et longue résistance en Russie ${ }^{33}$. Ces circonstances

p. 24 sq. Ces premiers débats ont été renforcés de quelques démonstrations nouvelles et republiés dans M. David-Fox, P. Holquist, A. M. Martin, Orientalism and Empire in Russia. Egalement, A. S. Morrison, “Applied Orientalism” in British India and Tsarist Turkestan".

29. L. Wolff, Inventing Eastern Europe; M. Poe, A People Born to Slavery. Rappelons que la langue russe continue à être enseignée à Paris à l'Institut national des langues et des civilisations orientales (INALCO).

30. M. Bassin, "Eurasianism "Classical” and "Neo" ; M. Laruelle, Russian Eurasianism.

3I. Voir, p. ex., O. Figes, Natasha's Dance.

32. Alors que le KGB l'avait rapidement traduit pour son usage interne (information de Stéphane Dudoignon, juin 2011) et la revue Kino [Cinéma] a publié quelques passages en 1995-2003, la version complète pour grand public ne parut qu'en 2006. De qualité médiocre, cette traduction a par ailleurs été accaparée par les droites nationalistes russes qui ont présenté - par la voix de Konstantin A. Krylov, auteur de la préface à cette traduction, la Russie comme la première victime de l'orientalisme occidental.

33. Sur la réception de ce livre dans le monde postsoviétique voir V. O. Bobrovnikov, "Počemu my marginaly?", ainsi que la contribution de D. Schimmelpenninck van der Oye dans le présent volume; par contraste, pour l'analyse d'une expérience personnelle - négative - de la prise de connaissance des études postcoloniales voir aussi S. Serebriany, "Comparative Literature and Post-colonial Studies». Par rapport à l'Inde et à la Russie, il est par ailleurs intéressant de souligner la ressemblance d'un grand nombre des arguments avancés par les détracteurs des approches saïdiennes. Pour une dernière analyse de l'héritage de Saïd, voir D. M. Varisco, Reading Orientalism, ainsi que la critique farouche lancée par J.-F. Bayart, Les études postcoloniales. 
ne parviennent toutefois pas à empêcher l'introduction de cette aire dans le champ des études postcoloniales. Partant de leur propre documentation, les russisants ${ }^{34}$ commencent à s'associer prudemment - car venus plus tard - au réexamen des approches postcoloniales dont le champ d'investigation s'est également élargi à de nouveaux horizons comme ceux de l'Allemagne, de l'Italie, du Japon ou de l'Europe centrale, analysés à l'aune de la problématique des empires ${ }^{35}$. Par ailleurs, cette arrivée tardive dans la problématique postcoloniale fait que dans les années 2000 les polémiques entre russisants se présentent de manière plus nuancée en comparaison de la situation que la communauté des spécialistes de l'Inde et du Moyen-Orient connaît dans les années 19801990.

Tout en respectant la distinction entre les époques tsariste et soviétique à travers la rhétorique de la continuité et de la rupture, divers

34. Une partie de ces chercheurs œuvrant dans les domaines connexes aux études postcoloniales est constituée par des spécialistes d'origine soviétique émigrés en "Occident", ce qui souligne de nouveau, pour la formation de ce type d'approche et comme dans le cas de l'Inde, l'importance du milieu des intellectuels en exil, issu de la fuite des cerveaux subie par leur patrie d'origine.

35. Sans parler de la Grande-Bretagne et des Etats-Unis, toujours en tête des réflexions postcoloniales, l'engouement pour un réexamen des corrélations entre recherche et agendas politiques a touché plusieurs pays comme, entre autres, la France, la Russie, les Pays-Bas, l'Allemagne (S. Friedrichsmeyer, S. Lennox, S. Zantop, The Imperialist Imagination; Sh. Pollock, "Deep Orientalism?»; E. Ames, M. Klotz, L. Wildenthal, Germany's Colonial Pasts; U. Wokoeck, German Orientalism), l'Italie (P. Palumbo, A Place in the Sun; R. Ben-Ghiat, M. Fuller, Italian Colonialism; N. Vallorani, E. Monegato, Orientalism/Orientalisms) et le Japon (L. Young, Japan's Total Empire; A. Dudden, Japan's Colonization of Korea; H. Mizuno, Science for the Empire). Pour l'Europe centrale, voir la revue Postcolonial Europe: <http://www. postcolonial-europe.eu/>. Pour la France, on peut songer aux travaux relatifs aux "sciences coloniales» menés par des historiens tels que Claude Blanckaert, Daniel Nordman ou Kapil Raj, actifs au centre Alexandre Koyré du Muséum d'Histoire naturelle de Paris, ou tels qu'Hélène Blais, Armelle Enders, Jean-François Klein, Emmanuelle Sibeud, Pierre Singaravelou et Sylvie Thénault dans le cadre du séminaire «Empires. Histoires des colonisations» organisé à l'ENS. On peut également citer un programme dirigé par François Pouillon à l'EHESS sur les orientalistes de langue française (<http://dictionnairedesorientalistes.ehess.fr/document.php?id=35>) qui a débouché récemment sur un colloque important (F. Pouillon, J.-C. Vatin, Après l'orientalisme). Voir également en dernier lieu la présentation de l'histoire universelle à travers une synthèse portant sur les empires dans la longue durée, de l'Empire romain au $\mathrm{XX}^{\mathrm{e}}$ siècle, par J. Burbank, F. Cooper, Empires in World History, ainsi que les réflexions de F. Cooper, A. L. Stoler, Tensions of Empire, p. 1-56. 
séminaires et colloques ${ }^{36}$ sont très rapidement - en une décennie - parvenus à proposer avec succès une application à l'histoire russo-soviétique des modèles et théorisations élaborés sur la base des exemples des puissances occidentales (l'analyse littéraire n'a en revanche pas atteint le même niveau d'attention que celui qui, entre autres, s'est manifesté en Inde). Thèses et publications de formats divers en ont alors constitué le débouché logique par un mélange à volonté des approches de type "nouvelle histoire impériale " ${ }^{37}$, «Imperial Turn» ${ }^{38}$, «études des nationalités / minorités» ou "impériologie» 39 .

Cet engouement pour les empires, les questionnements sur le colonialisme et le brassage des chercheurs dans les divers centres d'études liés à de larges programmes d'échanges sont en train de conduire à une «normalisation" de la période impériale de l'histoire russe, non seulement dans les traditions académiques occidentales, mais aussi, quoique dans une mesure bien moindre, dans le monde postsoviétique ${ }^{40}$. Le fait qu'une grande partie des mécanismes de soumission soit similaire dans tous les empires

36. Sans vouloir dresser un panorama exhaustif, rappelons que, sur des niveaux de pertinence différents, l'orientalisme russe a été récemment questionné lors de divers colloques à Moscou (Orientalism/Occidentalism: Languages of Cultures vs Languages of Descriptions, 23-25 septembre 2010, par Evgeny Steiner; Izučaja islam, izučaja drug druga: zapadnye $i$ sovetskie vzgljady na islam $v$ Central'noj Azii i na Kavkaze, 25-27 juin 2012 par Mikhael Kemper; Mir imperij: učenye, znanija $i$ «iskusstvo upravlenija" periferiej v kolonial'nyh i kontinental'nyh imperijah, 12-14 novembre 2012, par A. O. Cubar'jan et S. Karner), à Cambridge (Postcolonial Approaches to Postsocialist Experiences, 24-25 février 2012, par Alexander Etkind et Dirk Uffelmann), à Beyrouth (Russia's Orient, $1^{\mathrm{er}}-2$ juin 2012 par Paul du Quenoy), à Oxford (Russian Orientalism to Soviet Iranology, 30 novembre-1 ${ }^{\text {er }}$ décembre 2012, par Edmund Herzig et Stephanie Cronin), à Cambridge (Orientality: Cultural Orientalism and Mentality, 17-18 mai 2013, par Firuza Melville, Olga Nefedova, Jean Michel Massing), à Columbia University (Late Imperial Epistemologies: A Eurasian Studies Workshop, 17-18 mai 2013).

37. Voir les revues Ab Imperio et Kritika: Explorations in Russian and Eurasian History, travaillant sur de multiples aspects de l'empire russe.

38. M. David-Fox, P. Holquist, A. M. Martin, The Imperial Turn.

39. K. Matsuzato, Imperiology.

40. En addition au tour d'horizon des dernières publications sur l'expérience coloniale russe en Asie centrale in S. Gorshenina, «La marginalité du Turkestan colonial russe est-elle une fatalité?», p. 45-57, voir l'analyse très détaillée des publications parues après 1990 à propos des périphéries orientales de l'empire russe: Sunderland, 2011. Egalement, la discussion faisant le bilan de ces dix ans de réflexions sur les empires dans la revue $A b$ Imperio: Forum $A I$, "The Imperial Turn in Russian Studies: Ten Years Later». 
des $\mathrm{XIX}^{\mathrm{e}}-\mathrm{XX}$ e siècles commence à être partout reconnu, même s'il est admis que l'empire russe puisse avoir eu quelques traits particuliers que l'on commence à mieux connaître. En évitant de désigner des exemples «classiques» et d'uniformiser les pratiques coloniales de l'empire, l'analyse renouvelée de ces phénomènes accorde une priorité particulière à la diversification des regards posés sur les multiples situations coloniales dans les «Orients» russes. Ces situations sont désormais contextualisées selon de fines tranches chronologiques et régionales en fonction de la particularité culturelle des peuples soumis à la juridiction russe ${ }^{41}$.

En poussant plus loin, des outillages intellectuels postcoloniaux ont été également appliqués à l'analyse des relations entre les élites et le peuple (essentiellement paysan) russes, imbriquant l'histoire russe dans un système de réflexions propres à un orientalisme social. Par rapport à la Russie, ce dernier se présente cependant sous le terme "orientalisme de l'intérieur" 42.

Renforçant ces études historiques «impériales», plusieurs livres publiés coup sur coup dernièrement en Europe et en Amérique du Nord ont été consacrés à l'orientalisme russe dans le sens le plus proche de celui qui a été désigné par Saïd, notamment sur la production des connaissances et des représentations. De nature panoramique, les ouvrages de Lorraine de Meaux et de David Schimmelpenninck van der Oye brossent en français et en anglais des tableaux assez semblables, mais complexes où

4I. Voir, p. ex., les variations notables que l'on perçoit dans le cadre de la colonisation des espaces périphériques russes: N. B. Breyfogle, A. Schrader, W. Sunderland, Peopling the Russian Periphery. Il est également intéressant de souligner sur l'exemple de l'Asie centrale comment se profilent actuellement les directions d'études qui visent à définir dans le cadre colonial des éléments de l'histoire sociale (J. Sahadeo, Russian Colonial Society in Tashkent; A. S. Morrison, Russian Rule in Samarkand) ou des notions comme celle de la propriété (N. Pianciola, Stalinismo di frontiera; P. Sartori, "Introduction»). Pour un recueil d'articles essayant explicitement d'appliquer les méthodes des analyses postcoloniales à l'histoire du Turkestan russe voir également S. Gorshenina, S. Abashin, Le Turkestan russe. Inscrit dès le départ dans l'agenda de la recherche postcoloniale, le point de vue local sur les événements coloniaux a été également reconnu dans toute son importance; dans le cadre de ce type d'analyse, voir notamment A. Erkinov, Praying for and against the Tsar; B. M. Babadžanov, Kokandskoe hanstvo, ainsi que le workshop organisé par Ulfat Abdurasulov et Thomas Welsford, New Approaches in Islamic-, Tsarist-and Soviet-Era Central Asian History (ca. 750-1991), à Tachkent, les 25-26 septembre 2013.

42. Plusieurs publications d'Alexandre Etkind traitent de ce sujet; voir notamment A. Etkind, Internal Colonization; A. Etkind, D. Uffelmann, I. Kukulin, Tam, vnutri. 
l'orientalisme est présenté à la fois comme une tradition académique, un ensemble de techniques politiques, ou une série d'interprétations littéraires, théâtrales, musicales ou artistiques du thème de l' "Orient» ${ }^{43}$. Finalement, aux confins de ces multiples "Orients russes", créés dans une hybridité et une mixité des pensées et valeurs occidentales et orientales, c'est l'identité russe qui se dessine en filigrane au carrefour des discussions sur les empires, le colonialisme et l'orientalisme.

Mais le véritable bouleversement dans la vision de l'orientalisme russe et dans la genèse de l'orientalisme de Saïd en général se produit dans le livre Russia's Own Orient où Vera Tolz dresse les parallèles entre les orientalistes russes relevant de l'école du baron Viktor R. Rozen (18641908) et la théorie de Saïd ${ }^{44}$. En reconstruisant le chemin par lequel les approches critiques anti-impérialistes russo-soviétiques se sont transmises à Saïd par l'intermédiaire d'intellectuels marxistes arabes ${ }^{45}$, Tolz propose de considérer que c'est en Russie qu'on peut placer le foyer initial de la critique anticolonialiste, tout en soulignant la diversité des origines des chercheurs de l'orientalisme académique russo-soviétique (rappelons que l'origine des orientalistes anglophones ou francophones connaît elle aussi la même diversité). Profondément modelé par les savoirs occidentaux, mais en même temps composé de plusieurs acteurs d'origine "orientale», cet orientalisme est paradoxal et complexe, à l'image de la conquête russe, avec pour résultat la création du "propre Orient» de la Russie. Cet orientalisme tire profit tant de la politique expansionniste russe, qu'il critique et suit tout à la fois, que de la proximité unique des «objets d'études»

43. L. de Meaux, La Russie et la tentation de l'Orient; D. Schimmelpenninck van der Oye, Russian Orientalism. Rappelons cependant que des réinterprétations des motifs de l'«Orient» dans les arts russes ont été largement traitées avant et après la parution de ces livres. Voir, p. ex., R. Comtet, Les Russes et l'Orient; A. Ferrari, La foresta e la steppa; J. A. Sharp, Russian Modernism between East and West; A. Pondopoulo, Les Orients dans la culture russe. Du côté russe où l'approche est bien moins globale, la thèse d'Elena Čač (2012) ne traite que l'orientalisme russe, essentiellement littéraire, durant le siècle d'Argent. Voir également le catalogue de l'exposition Russia's Unknown Orient: Orientalist Painting 1850-1920 (P. Wageman, I. Kouteinikova, Russia's Unknown Orient).

44. V. Tolz, Russia's Own Orient. Devenu aussitôt un livre à succès, cette publication a non seulement bénéficié de plusieurs comptes-rendus, mais a suscité également une discussion intéressante dans la revue Ab Imperio: Forum AI, "Obsuždenie knigi Very Tolz".

45. A. Abdel-Malek, "L'orientalisme en crise». 
par rapport auxquels il joue un rôle actif dans une (ré)organisation des périphéries orientales de l'empire russo-soviétique tout en continuant à hésiter entre l'affirmation d'une suprématie russe et une discrimination positive à l'encontre des minorités.

En tant que composante la plus spécifique de l'orientalisme russe, l'orientalisme soviétique présente davantage le caractère d'une notion sujette à caution. Dans une mesure plus importante que pour l'époque tsariste, l'analyse de l'expérience soviétique dans l'optique des théories postcoloniales ${ }^{46}$ aboutit à des résultats encore plus paradoxaux et souvent contradictoires entre eux. D'une part, la fissure séparant sur ce sujet les spécialistes occidentaux, "vaccinés» depuis trente ans par les approches post-saïdiennes devenues finalement «traditionnelles» chez les jeunes chercheurs, et les scientifiques postsoviétiques, héritiers de l'isolationnisme des études orientales soviétiques, est à quelques exceptions près encore intacte. D’autre part, l'opinion occidentale est nuancée selon que l'accent est mis sur le caractère colonial pratiquement "classique" de l'URSS ${ }^{47}$ ou sur le potentiel mobilisateur soviétique et les larges programmes de modernisation tant culturelle qu'économique, lancés à travers la campagne de korenizacija et supportés par des injections budgétaires massives qui se sont étendues largement au-delà des années $1920^{48}$.

Parmi les exemples emblématiques de la complexité de ce type d'analyse se trouve le cas de l'Asie centrale - le plus «colonial»-, qui

46. Parmi les travaux les plus conséquents, le programme «Legacy of Soviet Oriental Studies: Networks, Institutions, Discourses" de l'Université d'Amsterdam (2009-2013), codirigé par Michael Kemper et Stéphane A. Dudoignon, a été consacré à l'analyse de l'orientalisme soviétique et postsoviétique afin d'inscrire ce cas dans la typologie des orientalismes européens: c'est dans ce cadre que plusieurs thèses ont été notamment lancées sur des sujets comme l'orientalisme soviétique à Leningrad et à Almaty (Alfrid K. Bustanov; thèse soutenue), les études orientales et le nationalisme en Azerbaïdjan (Sara H. Crombach), les institutions orientalisantes de Moscou et de Leningrad (Hanna E. Jansen). Pour les résultats publiés voir M. Kemper, S. Conerman, The Heritage of Soviet Oriental Studies. Voir également les réflexions d'Adeeb Khalid, "Backwardness and the Quest for Civilization», en introduction à un numéro spécial du Central Asian Survey consacré à ce sujet.

47. J. B. Baberovskij, Vrag est' vezde; R. P. Geraci, Window on the East; F. Hirsch, Empire of Nations; P. Michaels, Curative Powers; D. Northrop, Veiled Empire; K. Olofsson, From Orientalism to Postcoloniality; K. Sahni, Crucifying the Orient; E. Thompson, Imperial Knowledge.

48. T. Martin, The Affirmative Action Empire; A. Khalid, «Introduction». 
illustre finalement combien on ne peut procéder utilement à une application mécanique des théories postcoloniales à l'histoire soviétique tant la modernisation et la rhétorique anticoloniale/ anti-impérialiste se mêlent aux pratiques coloniales, notamment aux transformations sociales forcées. Cela est d'autant plus vrai que de nombreux orientalistes soviétiques sont eux-mêmes issus de contextes "périphériques" comme l'Asie centrale ou le Caucase. Ces faits n'interdisent cependant plus de considérer que le processus d'écriture des histoires nationales mis en place dans chacune des républiques méridionales nouvellement créées lors de la délimitation nationale de 1924-1936 relève d'objectifs politiques et, donc, d'un phénomène d'"orientalisation". Il en va de même pour l'activité d'expertise des orientalistes soviétiques sur le sujet de l'Islam, que ce dernier soit «domicilié» en URSS ou qu'il vienne de l'étranger.

Les hésitations sur la nature - coloniale ou postcoloniale - du pouvoir soviétique influencent également les débats portant sur la question de savoir si l'époque postsoviétique est ou non à considérer comme une période postcoloniale ${ }^{49}$, alors même que plusieurs caractéristiques autorisent à inclure les républiques ex-soviétiques dans la catégorie du «Sud » propre au tiers-monde postcolonial ${ }^{50}$.

Force est de constater, finalement, que ce qui dans l'histoire soviétique ou postsoviétique est (post)colonial ou post-postcolonial reste toujours difficile à cibler et à analyser. Cependant, on peut dire que les griffes de ce type d'approches ont bien pris dans l'historiographie contemporaine des études russo-soviétiques. L'opinion selon laquelle il importe de continuer à réfléchir dans cette direction afin de combler la lacune historiographique subsistant à côté des lectures marxistes, nationalistes et impérialistes du passé russo-soviétique, regagne du terrain ${ }^{51}$; le travail

49. D. Kandiyoti, «Post-Colonialism Compared»; L. Adams, "Can we Apply Postcolonial Theory to Central Eurasia?»; M. Laruelle, «Le paradigme du colonialisme en Asie centrale postsoviétique»; A. Remnev, "Kolonial'nost', postkolonial'nost' i "istoričeskaja politika” v sovremennom Kazahstane»; S. N. Abašin, «Nacii i postkolonializm v Central'noj Azii dvadcat' let spustja»; S. N. Cummings, R. Hinnebusch, Sovereignty after Empire.

50. Voir les résultats du colloque "The Caucasus and Central Asia, Twenty Years after Independances", Almaty, 25-27 août 2011: <http://lodel.ehess.fr/cercec/ docannexe.php?id=1705>.

5I. Voir l'analyse par Sergej N. Abašin "Zavoevanie Srednej Azii» de ces trois types d'interprétations de la conquête du khanat de Kokand - impérialiste pour M. Terent'ev, historien officiel de la conquête russe de l'Asie centrale, marxiste pour 
d'analyse critique qui a déjà été accompli pour l'Inde n'a en effet toujours pas abouti dans l'aire postsoviétique.

Mais pour que toutes les démarches soient respectées dans leurs justes proportions, il faut reconnaître que l'orientalisme russo-soviétique (ainsi que l'éventuel occidentalisme russe, centrasiatique, "caucasien", etc.) reste globalement un sujet marginal chez les spécialistes du monde russo-soviétique et postsoviétique et que les chercheurs sont loin d'avoir formulé une doctrine historiographique cohérente par rapport à cette problématique.

3.2. Inde et orientalisme: une problématique à plusieurs dimensions

L'Inde, quant à elle, s'est présentée dès l'inauguration des études postcoloniales comme un exemple paradigmatique, incarnant particulièrement bien la problématique saïdienne et susceptible d'expliquer la nature du colonialisme britannique. L'une des sources importantes de Saïd est d'ailleurs un ouvrage centré sur l'histoire des perceptions de l'Inde en Europe, la Renaissance orientale de Raymond Schwab ${ }^{52}$. Dès la parution de l'Orientalisme, plusieurs auteurs ont travaillé à partir de cette perspective sur différents cas de la période coloniale indienne. Ces travaux s'intéressent particulièrement à la collusion entre les projets savants européens et les intérêts coloniaux britanniques et insistent sur le fait que certaines caractéristiques majeures de la société indienne se sont développées au gré des intérêts de la classe dominante britannique. Par exemple, dans une collection d'articles publiée en 1987 et qui a fait date, l'anthropologue américain Bernard Cohn (1928-2003) démontre que la présence britannique a eu pour effet d'engendrer tacitement des changements socio-politiques majeurs au niveau local. Un élève de Cohn,

le professeur moscovite N. S. Kipjanina et nationaliste pour l'historien ouzbek de l'époque d'indépendance Kh. Ziëev. Pour illustrer la complexité des réflexions voir également, S. N. Abašin et al., Central'naja Azija v sostave Rossijskoj Imperii; S. N. Abašin, "Razmyšlenija o "Central'noj Azii v sostave Rossijskoj imperii" ".

52. R. Schwab, La renaissance orientale. Bien que Saïd en ait préfacé la traduction anglaise, l'ouvrage de Schwab ne porte pas la même attention que Saïd aux enjeux de pouvoir et se focalise sur le rôle de la "redécouverte" de la culture indienne et du sanscrit, qui constitue en quelque sorte une «seconde Renaissance» dans la construction de la modernité européenne. Sur les racines indiennes de la thèse de Saïd, voir D. Smith, «Orientalism and Hinduism». 
Nicholas Dirks, a poursuivi cette approche en soulignant l'impact décisif des Britanniques sur l'institution des "castes» dans sa configuration moderne ${ }^{53}$.

Dans une perspective similaire, la question de la transposition de catégories culturelles construites par l'administration coloniale ou les savants européens en réalités socio-politiques a également été abordée par rapport à de nombreuses dimensions de la société indienne. Parmi celles-ci, on peut mentionner les sujets de la langue (statut par exemple de l'anglais ou du hindi) ${ }^{54}$, des pratiques médicales ${ }^{55}$, des habits ${ }^{56}$, ou encore des traditions religieuses de l'Inde elles-mêmes, fréquemment représentées à partir d'un imaginaire christiano-centré 57 . Ces travaux se sont souvent montrés très critiques envers l'histoire des études indiennes elles-mêmes, soupçonnées de défendre, volens nolens, une idéologie foncièrement impérialiste. Ronald Inden propose ainsi une lecture de l'histoire de l'indologie à la lumière de la critique de Saïd. Il dénonce en particulier la focalisation des études sur les "castes», la religion hindoue, les villages et la figure du souverain. Cette focalisation trahit avant tout la prédominance de certains paradigmes qui trouvent leur raison d'être dans le contexte occidental seulement. Pour Inden, les travaux qui s'inscrivent dans ce contexte ne se servent de l'Inde que comme un prétexte, et relèguent les acteurs indiens eux-mêmes dans un rôle fondamentalement passif. Comme le montre Trautmann, cette perspective est cependant elle-même problématique, avant tout parce qu'Inden sélectionne

53. B. Cohn, An Anthropologist among the Historians and Other Essays; N. Dirks, Castes of Mind. Voir la critique de W. Pinch à l'égard de Cohn, "Review: Same Difference in India and Europe». W. Pinch lui-même n'échappe cependant pas à un certain irénisme dans sa description des contacts entre Britanniques et Indiens (voir à ce propos S. Subrahmanyam, «Par-delà l'incommensurabilité», p. 43-45).

54. B. Cohn, "The Command of Language and the Language of Command"; D. Lelyveld, "The Fate of Hindustani» et "Colonial Knowledge and the Fate of Hindustani»; G. Ch. Spivak, "The Burden of English».

55. D. Arnold, Colonizing the Body et Science, Technology and Medicine in Colonial India.

56. B. Cohn, "Cloth, Clothes, and Colonialism».

57. Ph. Almond, The British Discovery of Buddhism; D. S. Lopez, Curators of the Buddha (pour le bouddhisme); S. N. Balagangadhara, "The Heathen in his Blindness... "; Sh. Sugirtharajah, Imagining Hinduism (pour l'hindouisme). L'ouvrage de R. King, Orientalism and Religion, montre de quelle manière les "orientalisteshistoriens des religions" ont contribué à élaborer un "Orient mystique » à l'image de leurs propres conceptions religieuses. 
des travaux ne relevant pas de l'indologie «savante» et ne prend pas du tout en compte le rôle des savants indiens dans le processus de création de savoirs ${ }^{58}$.

En parallèle à ces travaux, de nouvelles perspectives font leur apparition dans le courant des années 1990. Ainsi, l'ouvrage collectif édité par Carol Breckenridge et Peter van der Veer réunit aussi bien des contributions prolongeant dans les grandes lignes le projet saïdien que des travaux problématisant cette approche ${ }^{59}$. Il semble que les réserves formulées de bonne heure à l'égard de l'Orientalism ${ }^{60}$ ont rencontré un terreau particulièrement fertile dans le contexte indien.

Il y a en premier lieu la question de la temporalité. L'orientalisme saïdien démarre vers la fin du XVIII ${ }^{\mathrm{e}}$ siècle environ, ce qui correspond pour l'Inde à la fondation de l'Asiatic Society à Calcutta (1784). Si cette période semble tout indiquée pour démontrer le lien entre le pouvoir colonial et les institutions savantes, plusieurs auteurs récents ont souligné l'importance d'examiner l'histoire coloniale indienne en regard de ce qu'on peut nommer la "préhistoire" de l'orientalisme. Pour bien comprendre les enjeux spécifiques à l'orientalisme (et plus particulièrement aux études indiennes) de l'époque coloniale, il est en effet nécessaire de disposer d'une image précise de la période qui précède - au moins de l'Inde moghole - et de la place des travaux savants (par exemple, ceux des Jésuites) dans ce cadre ${ }^{61}$. Le risque est sinon de tomber dans un idéalisme "naïf" par rapport à la situation précoloniale et de postuler une tabula rasa à l'arrivée des Britanniques ${ }^{62}$.

58. R. B. Inden, Imagining India. Voir les notes critiques de Th. R. Trautmann, Aryans and British India, p. 23 sq.

59. C. A. Breckenridge, P. van der Veer, Orientalism and the Postcolonial Predicament.

6o. P. ex., J. Clifford, "Review of Edward Said's Orientalism» ou A. Ahmad, "Orientalism and After». Plus récemment, on peut mentionner les ouvrages de J. M. MacKenzie, Orientalism: History, Theory and the Arts et de R. Irwin, For the Lust of Knowing dont les arguments critiques trouvent également des échos dans le contexte indien.

6r. Sur les enjeux de l'étude de la "préhistoire de l'orientalisme», voir Sh. Pollock, «Deep Orientalism?», p. 96-111 («pre-orientalist Orientalism»); W. Sweetman, «The Prehistory of Orientalism » et S. Subrahmanyam, "An Eastern El-Dorado " (en particulier, p. 382).

62. La question de la continuité entre les périodes précoloniale et coloniale est au cœur de l'ouvrage de Ch. A. Bayly, Indian Society and the Making of the British Empire. 
De manière spécifique à l'Inde, la définition même de l'orientalisme saïdien, associant en son sein les niveaux "populaire" et "savant", est apparue comme problématique pour plusieurs auteurs. Ceux-ci questionnent l'opportunité de mettre sur un pied d'égalité les usages politiques et philosophiques de l'Inde (chez J. Mill [1773-1836] ou G. W. F. Hegel [1770-1831] par exemple) avec les productions savantes issues des écoles indologiques (celles de Sir William Jones [1746-1794] ou de Horace Hayman Wilson [1785-1860] par exemple). Une frontière est ainsi tracée entre une perspective savante, informée et ancrée dans la "science indigène», et une perspective travaillant sur des sources de seconde voire de tierce main, et plus volontiers sujette aux processus d'instrumentalisation politique. David Kopf fut l'un des premiers à soulever ce point dans les années 1980, en montrant de quelle manière certains "orientalistes" britanniques comme Jones ou Wilson ne partageaient pas le même agenda que les autorités coloniales et y étaient même, dans certains cas, farouchement opposés ${ }^{63}$. De même, pour Thomas Trautmann, il s'agit de problématiser la nature des liens entre ces deux sortes d'orientalisme et non de partir d'une pétition de principe énonçant leur identité ${ }^{64}$.

63. Cf. D. Kopf, «Hermeneutics versus History», au sujet de l'administration britannique dans le contexte bengali des premières décennies du XIX ${ }^{\mathrm{e}}$ siècle. Pour Kopf, les «orientalistes» défendent une position contraire à celle des «anglicistes» dans la controverse qui les oppose. Un grand artisan de l'«anglicisation» fut Thomas B. McCaulay (1800-1859) dont les propos méprisants à l'égard de la civilisation indienne restent amèrement remémorés en Inde. En ce sens, les "orientalistes", fervents défenseurs de l'enseignement des langues orientales (sanscrit, persan, etc.) et opposés à l'idée d'anglicisation de la société indienne ne correspondent pas bien à l'image des "orientalistes» de Saïd. Voir également R. Rocher, "British Orientalism in the Eighteenth Century", et son analyse de la complexité des relations entre les "orientalistes savants» et les autorités coloniales.

64. Th. R. Trautmann, Aryans and British India, distingue différents types de savoirs orientalistes en fonction de la connaissance des langues et du rapport à la «science indigène». Déjà S. Lévi, "Les parts respectives des nations occidentales dans les progrès de l'indianisme», p. 110, notait que "[1]e travail proprement scientifique reste sous l'influence de la science indigène, et [...] les spéculations d'ordre livresque se corrigent d'elles-mêmes par l'impression directe des réalités ». L'ouvrage de R. Lardinois, L'invention de l'Inde, propose une analyse nuancée et problématisée des liens entre les travaux savants et les productions qui ne témoignent pas d'une connaissance intime (linguistique) des contextes. 
Une autre réserve formulée à l'égard de Saïd - sa focalisation sur les écoles anglaise et française, à l'exclusion partielle d'autres traditions savantes - trouve également un écho dans les études récentes des travaux orientalistes sur l'Inde. Certains ont fait remarquer toute l'importance des traditions indologiques issues d'autres contextes nationaux que la France ou l'Angleterre, qui entretiennent un rapport plus complexe (et plus ambigu) aux entreprises impériales et coloniales. Un autre argument allant dans la même direction consiste à interroger la notion même d' "école nationale", en prenant en compte la question de la circulation des savants, non seulement entre différentes institutions européennes mais aussi entre l'Europe et l'Inde ${ }^{65}$.

Enfin, deux questions liées, également en débat avec Saïd, sont au centre de nombreux travaux récents: les contacts entre les indologues et les élites indiennes d'une part, et la réception des travaux orientalistes en Inde d'autre part. Contre l'idée d'une césure radicale entre les orientalistes et l'objet même de leurs études, plusieurs auteurs insistent aujourd'hui sur les liens qui les unissent. Sur ce sujet, un ouvrage pionnier est celui de Wilhelm Halbfass, India and Europe (1981), qui retrace l'histoire des relations entre Inde et Europe de l'époque antique jusqu'au $\mathrm{XX}$ siècle et qui curieusement (ou stratégiquement) ne cite Saïd à aucun moment. Dans le sillage de cette perspective, on pourra alors revisiter les biographies de grandes figures d'orientalistes, en s'intéressant également à leur rôle de "passeurs" et aux contacts qu'ils ont tissés, à la fois dans leur contexte académique européen et en Inde. Ainsi, Sir William Jones dut la plus grande partie de ses connaissances de la langue sanscrite et des textes sanscrits à ses pandits ${ }^{66}$. De même Friedrich Max Müller

65. Voir p. ex. P. Rabault-Feuerhahn, "Voyages d'études et migrations savantes», qui insiste sur la circulation des savants indologues dans l'Europe du XIX ${ }^{\mathrm{e}}$ siècle. Dès le milieu du XIX ${ }^{\mathrm{e}}$ siècle, de nombreux indologues engagés par des institutions britanniques seront bien souvent des Allemands, qui auront eux-mêmes étudié en plusieurs lieux (notamment à Paris, chez E. Burnouf [1801-1852]). Pour un exemple de circulation des connaissances qui échappe à toute notion d'école nationale, voir U. App, «How Amida Got into the Upanishads». Sur l'indologie allemande, voir p. ex. Sh. Pollock, German Orientalism, et A. Choné, Rudolf Steiner, Carl Gustav Jung, Hermann Hesse. L'article de C. Regamey, "Un pionnier vaudois des études indiennes», demeure une excellente introduction à l'histoire des études indiennes en Suisse, discipline difficilement rattachable à une "école nationale».

66. Sur les pandits de W. Jones, voir A. Mukerji, «European Jones and Asiatic Pandits»; R. Rocher, "The Career of Rādhākānta Tarkavāgīśa» et M. Dodson, 
(1823-1900), souvent considéré comme la figure archétypique de l'orientaliste occidental dans sa tour d'ivoire, entretenait une correspondance avec plusieurs savants indiens et ses travaux ont été bien reçus en Inde ${ }^{67}$. Ces cas peuvent être fructueusement étudiés dans le cadre d'une histoire "connectée", comme dans certains travaux de Sanjay Subrahmanyam relisant les parcours de savants orientalistes à la lumière d'une double contextualisation, à la fois au niveau européen et au niveau indien ${ }^{68}$.

Ainsi, dans ses travaux sur les missions chrétiennes en général et la notion d' "hindouisme» en particulier, David Lorenzen insiste sur l'idée d'une "collaboration" entre Indiens et Européens, critiquant du même coup la thèse d'une "invention" purement occidentale de la notion ${ }^{69}$. Il ajoute que l'application stricte d'une approche saïdienne au cas indien a pu involontairement contribuer à la marginalisation de certains acteurs indiens ayant interagi avec les Européens. Similairement, dans son étude de l'histoire du royaume rajasthani de Koțā, Norbert Peabody met l'accent sur le caractère imbriqué des relations entre Européens et Indiens ${ }^{70}$. Plaidant pour une construction "en dialogue» de l'histoire, Eugene Irschick a défendu une position tout à fait similaire à partir de son étude

Orientalism, Empire, and National Culture. La revue Asiatic Researches fondée par Jones publia des contributions de certains de ces érudits et ces derniers purent devenir membres à part entière de l'Asiatic Society du Bengale qui ouvrit ses portes aux Indiens en 1829, à l'instigation de l'indologue britannique Horace H. Wilson.

67. Cf. C. J. G. Van der Burg, "The Place of Sanskrit in Neo-Hindu Ideologies», p. 370-373, qui note, p. ex., qu'Aurobindo (1872-1950) fut en contact direct avec Müller lors de ses études en Angleterre (1879-1892) et dut lire le texte de Müller, India, What Can It Teach Us (1882) dans le cadre de sa formation de "Civil Servant». Sans doute marqué par ces expériences, Aurobindo reprit des éléments de la rhétorique "aryaniste» dans ses propres travaux. Dans un registre similaire, les conclusions de Müller en linguistique historique furent largement saluées en Inde: celles-ci permettaient de réduire l'écart entre les Indiens et leurs maîtres britanniques, puisqu'il leur était supposé «un glorieux ancêtre commun" (voir Th. Raychaudhuri, Europe Reconsidered, p. 8).

68. Voir, p. ex., S. Subrahmanyam, "Monsieur Picart and the Gentiles of India» et "The Career of Colonel Polier and Late Eighteenth-Century Orientalism», sur respectivement B. Picart (1673-1733) et A.-L. de Polier (1741-1795).

69. Cf. D. N. Lorenzen, "Who Invented Hinduism?", p. 639, qui note: «If Hinduism was invented, it was invented by European and Indian scholars working in tandem".

70. N. Peabody, Hindu Kingship and Polity in Precolonial India. 
de l'histoire du Sud de l'Inde aux XVIII ${ }^{\mathrm{e}}$ et XIX siècles $^{71}$. En extrapolant cette observation au cas russo-soviétique, soulignons quactuellement une attention particulière est également portée sur le rôle actif des acteurs locaux aussi bien au niveau des décisions politiques que lors de l'élaboration de réflexions scientifiques ${ }^{72}$.

Par ailleurs, dans une perspective centrée sur les contextes locaux, de nombreux travaux font aujourd'hui porter leur analyse non pas sur les figures bien connues d'orientalistes ou indologues européens, mais au contraire sur certains acteurs indiens - pour certains déjà étudiés par ailleurs et pour d'autres restés jusqu'à présent dans l'ombre ${ }^{73}$. Il s'agit de montrer que les élites indiennes ne sauraient être considérées comme passives et que les travaux européens ont fait l'objet de réactions, d'appropriations ou de rejets divers ${ }^{74}$. Il devient alors possible de parler d'un "orientalisme indien" à part entière, qui se rapporte en premier lieu à l'étude académique des cultures de l'Inde en Inde. A cet égard, l'histoire de la collecte de manuscrits entreprise au Maharashtra, sous la direction des indologues allemands G. Bühler (1837-1898) et F. Kielhorn (18401908) est tout à fait parlante. Ceux-ci développèrent leur activité à partir de Mumbai et Pune respectivement, et formèrent de nombreux élèves indiens. L'un d'entre eux, le Sir Dr. Ramakrishna Gopal Bhandarkar (1837-1925) obtiendra un doctorat de l'université de Göttingen et fait figure de l'un des premiers "orientalistes indiens" au sens strict du terme ${ }^{75}$.

7I. Cf. E. F. Irschick, Dialogue and History, p. 8, qui note: «[My work] suggests that changed significations are the heteroglot and dialogic productions of all members of any historical situation, though not always in equal measure».

72. A. Khalid, "Central Asia between the Ottoman and the Soviet Worlds".

73. P. ex., l'ouvrage de E. Leach, S. N. Mukherjee, Elites in South Asia.

74. Voir à ce propos Th. Raychaudhuri, Europe Reconsidered; Th. R. Trautmann, Aryans and British India, p. 218-221; K. Chatterjee, Europe Observed. A. S. Morrison, " "Applied Orientalism” in British India and Tsarist Turkestan», p. 624-627, développe l'expression d'"orientalisme oriental", tandis que F. Pouillon et J.-C. Vatin, Après l'orientalisme, utilisent l'expression «l'Orient créé par l'Orient». Pour une analyse de ce phénomène à partir de l'exemple d'artistes de l'Asie centrale d'origine locale investis dans la production orientaliste voir également S. Goršenina, B. Čuhovič, "Srednjaja Azija kak fenomen čistogo orientalističeskogo èksperimenta (1860-1990-e gg.)».

75. C'est en son honneur que sera créé en 1917 le Bhandarkar Oriental Research Institute de Pune, où sont conservés jusqu'à aujourd'hui une grande partie des manuscrits collectés par Bühler et Kielhorn. Cet institut est à l'origine d'une édition critique du Mahābhārata. Le Deccan College de Pune, lui aussi une institution établie de 
Dans un sens plus métaphorique (mais conforme à l'acception saïdienne du terme), l'« orientalisme indien" peut désigner les représentations (artistiques, philosophiques, populaires, etc.) de l'Inde élaborées en Inde, par contraste avec ou en référence à l'Europe. Ainsi, par exemple, de l'écriture de l'histoire indienne, qui peut s'apparenter à une forme d' "orientalisme à rebours", en présentant un jeu subtil entre rejet de conceptions européennes de l'Inde perçues comme négatives ou offensives, et appropriations de pratiques savantes, conventions discursives, distinctions conceptuelles, etc. ${ }^{76}$. Dans ce cadre, on pourra songer à la Greater India Society (notamment les travaux de l'historien Ramesh Chandra Majumdar [1888-1980]) qui développe sa vision de l'histoire indienne en effectuant de larges emprunts à la tradition "orientaliste» européenne ${ }^{77}$ ou encore à l'ouvrage classique de Nehru, The Discovery of India ${ }^{78}$, qui réagit fortement à une conception britannique de l'histoire indienne. L'étude de l'historiographie indienne rencontre un intérêt croissant aujourd'hui, que ce soit dans une perspective purement historique ou pour réfléchir à des enjeux épistémologiques relatifs à l'écriture de l'histoire en général ${ }^{79}$.

longue date (1821), abrite quant à lui le projet de Thesaurus de la langue sanscrite (en cours).

76. A. Nandy, The Intimate Enemy, fait une bonne analyse de l'ambiguïté de ces processus d'intégration et d'appropriation. Un exemple particulièrement intéressant est celui des premières histoires de l'Inde écrites par des Indiens. Voir à ce propos l'exemple de la Rājābali (1808) de Mṛtyuñjay Vidyālañkār (1762-1819) (P. Chatterjee, "Alternative Nations, Alternative Histories», p. 231-236 et les remarques de B. Auer, infra p. 211) ou encore celui d'Ishvar Chandra Vidyasagar (1820-1891) et de son histoire du Bengale: Baíglär Itihās (1848) (W. Halbfass, India and Europe, p. 247).

77. Voir à ce propos S. Bayly, "Imagining "Greater India”"; C. Stolte, H. FischerTiné, "Imagining Asia in India», p. 82-88. Les savants de la Greater India Society s'efforcèrent de présenter l'Inde sous l'angle d'une force active dans l'histoire, ayant jadis colonisé et civilisé le Sud-Est asiatique (voire au-delà), en inversant la perspective britannique qui décrivait l'Inde sous un angle essentiellement passif. En éminents représentants de cette école, Kalidas Nag (1888-1980) et Prabodh Chandra Bagchi (1898-1956) ont eux-mêmes étudié à Paris, auprès de Sylvain Lévi et Jean Przyluski (1885-1944) et se sont approprié des conceptions telles que celle d'Inde civilisatrice (titre d'un ouvrage de $S$. Lévi) pour en livrer une réinterprétation à tendance nationaliste.

78. J. Nehru, The Discovery of India.

79. Voir, p. ex., dans des registres différents, R. Guha, History at the Limit of WorldHistory, P. Chatterjee, "Alternative Nations, Alternative Histories»; G. Prakash, «PostOrientalist Histories of the Third World»; D. Chakrabarty, Provincializing Europe; Th. R. Trautmann, «Does India Have History?». 
L'«orientalisme indien» peut aussi se référer à des mouvements qui réifient une notion comme celle d'"Orient" ou d' "Asie» dans un but essentiellement idéologique et politique. L'exemple de Rabindranath Tagore et de son "asiatisme" illustre bien ce point: Tagore cherche à promouvoir une tradition qu'il conçoit comme authentiquement asiatique pour laquelle il souhaite mobiliser un large nombre d'acteurs de pays asiatiques ${ }^{80}$. Il fonde son discours sur une opposition entre Asie et Occident, ce qui suppose la consistance et l'homogénéité de chacun de ces pôles dans leurs aspects culturels, économiques et surtout philosophiques. Il souscrit ainsi dans les grandes lignes à l'idée d'une "sagesse orientale» s'opposant au matérialisme incarné par l'«Occident» - un topos éculé de la vision européenne de l'Inde. Dans ce même ordre d'idées, des travaux récents cherchent à tracer les généalogies «orientalistes» d'idéologies politiques soutenues au sein de partis fédérateurs comme celui du Congrès ou de partis visant des intérêts plus particularistes, comme le Bharatiya Janata Party ou le Dravida Munnetra Kazhagam ${ }^{81}$.

$\mathrm{Au}$ vu de ces différents exemples, il semble que les perspectives récentes tendent à remettre en cause une approche de l'orientalisme qui procéderait par couples antinomiques (colon / colonisé, centre/ périphérie, Occident/ Orient, actif/ passif, etc.) et qui se limiterait à étudier sous un angle critique les parcours d'orientalistes occidentaux dans le contexte européen. La réelle complexité historique est ainsi plutôt décrite au moyen de théories de la circulation des idées - avec certes une prise en compte des asymétries et sans irénisme - que de schémas dichotomisants. C'est dans une telle perspective "connectée» que semblent se diriger aujourd'hui la plupart des travaux touchant à la thématique de l'orientalisme en lien avec l'Inde ${ }^{82}$.

De ce point de vue et de manière inattendue, l'Inde tout comme la Russie, se présente donc tout à la fois comme «objet» et comme «sujet» de l'orientalisme.

Ces deux trajectoires - russe et indienne - sont à première vue fort différentes tant à l'égard des histoires respectives des études orientalisantes,

80. Sur l'«asiatisme» de Tagore, voir S. N. Hay, Asian Ideas of East and West; C. Stolte, H. Fischer-Tiné, «Imagining Asia in India», p. 76-80.

8I. Voir, p. ex., P. van der Veer, "Hindu Nationalism and the Discourse of Modernity" et Religious Nationalism; A. Nandy, Creating a Nationality.

82. Voir ainsi entre autres, K. Raj, Relocating Modern Science; S. Sivasundaram, Nature and the Godly Empire et " A Christian Benares" ". 
que par rapport aux processus de l'orientalisation et de leur interprétation dans l'historiographie contemporaine, nationale et internationale, même s'il est possible de parler dans les deux cas d'une riche tradition savante locale, relevant d'un «orientalisme oriental». La comparaison d'aspects spécifiques de ces trajectoires à deux vitesses prend un relief particulier par une focalisation sur le phénomène des "marges», une notion longtemps délaissée au profit de celle du "centre». Loin de ne constituer que de simples "satellites" guidés par un "centre» ou une "métropole", les marges nous semblent au contraire donner les clés d'importants processus se déroulant au «centre».

\section{4. "Orientalisme des marges»: construire un comparable}

\subsection{Les marges peuvent être au centre}

Depuis une dizaine d'années, les chercheurs déplacent progressivement leur regard du "centre" vers les zones obscures de la périphérie, d'où l'intérêt qui est en train de se manifester pour les «marges». Cette approche finit d'ailleurs par occuper une position centrale dans les diverses problématiques nées aux carrefours interdisciplinaires. Travaillée de longue date par les anthropologues, à commencer par Georg Simmel (1858-1918) et Robert E. Park (1864-1944) ${ }^{83}$, la notion de "marges» est désormais bien apprivoisée dans le langage des chercheurs en sciences humaines, y compris chez les spécialistes de l'Antiquité ${ }^{84}$ ou les littéraires ${ }^{85}$. Elle ne répond toutefois pas à une clé de lecture unique, à l'image de la multitude des expressions symboliques, métaphoriques et épistémologiques relatives aux "marges».

83. Voir p. ex. R. Park, «Human Migration and the Marginal Man», et plus récemment la "théorie de la marginalité» d'A. Weisberger, "Marginality and its Directions".

84. Voir notamment E. S. Gruen, Rethinking the Other in Antiquity, chez qui l'histoire antique, associée au concept de l'«Autre», est présentée à travers l'histoire des marges des mondes grec et romain.

85. Comme exemple voir l'étude de S. Alatorre, "An Approach to Renaissance Margins", où sont analysés les espaces marginaux qui à l'époque de la Renaissance ont pris la forme métaphorique du labyrinthe. 
Malgré toute son ambiguïté, l'opposition spatiale "centrepériphérie " ${ }^{86}$ semble être plus communément utilisée dans les sciences sociales et humaines, notamment chez les politistes, géographes, urbanistes et économistes. Liée au concept des Etats-nations et de pouvoir, cette opposition tend à réduire la notion des "marges" à une notion d'«espace (semi-)périphérique».

L'histoire russo-soviétique-postsoviétique est à plusieurs égards facilement associable à cette acception des "marges", que ce soit par rapport à l'Europe occidentale, ou par rapport au fait que, tout en restant extrêmement centralisé, l'empire s'est étendu sur une aire très large à l'intérieur de ses frontières, au point qu'une partie a été sans ambiguïté qualifiée d'Extrême-Orient. Après un premier passage en revue de la construction et du fonctionnement des zones frontières de l'empire ${ }^{87}$ et des rapports de soumission (qu'elle soit coloniale ou liée à la centralisation soviétique), cette opposition "centre-périphérie» est surtout entrée dans l'analyse des processus de désintégration postsoviétique. L’accent est actuellement porté sur les migrations et les dépendances de tous types auxquelles sont confrontées les anciennes républiques par rapport à l'ex-métropole ${ }^{88}$. Sans quitter réellement leur statut dans les marges du système-monde, les nouveaux Etats s'efforcent de souligner le rôle clé qu'ils ont joué dans l'histoire universelle ${ }^{89}$ et tentent de s'intégrer, en tant qu'élément

86. Cf. pour une discussion théorique de cette opposition, l'excellent article d'A. de Toro, "Post-Coloniality and Post-Modernity».

87. R. P. Geraci, M. Khodarkovsky, Of Religion and Empire; M. Khodarkovsky, Russia's Steppe Frontier; B. J. Boeck, Imperial Boundaries; S. Gorshenina, Asie centrale. Pour un exemple hors du monde russo-soviétique mais utile pour le sujet voir F. Zarinebaf, J. Bennet, J. L. Davis, A Historical and Economic Geography of Ottoman Greece.

88. S. M. Birgerson, After the Breakup of a Multi-Ethnic Empire; M. Laruelle, S. Peyrouse, L'Asie centrale à l'aune de la mondialisation.

89. Cette demande, d'abord formulée par les Centrasiatiques, auteurs principaux de ce type de narrations, a été également entendue par des chercheurs et écrivains occidentaux comme le montre l'analyse de quelques récentes publications: J. Lally, «Going Global». En même temps, la notion des «marges», associées aux problèmes du sousdéveloppement et de l'enclavement, transparaît largement dans les publications traitant de l'histoire contemporaine de l'Asie centrale: M. Spechler, "Central Asia on the Edge of Globalization"; S. Tranum, Life at the Edge of the Empire; F. Mühlfried, S. Sokolovskiy, Exploring the Edge of Empire; E. Kazimzade, "In the Margins"; I. Silova, Globalization on the Margins; F. Wennberg, On the Edge. 
central, dans la mondialisation ${ }^{90}$; dans ce cas, il est possible de parler des «marges» comme d'une passerelle vers un pouvoir régional ou international qui, bien entendu, reste pour le moment à l'état de désir.

Si le monde indien, géographiquement plus compact, peut a priori sembler plus éloigné de cette problématique, la thématique des marges, considérée dans sa dimension géographique, sociale ou théorique a déjà fait l'objet de plusieurs analyses. Dans le contexte de l'Inde coloniale, et de manière classique, la notion de «marge" peut tout d'abord évoquer les acteurs indiens, qui furent souvent considérés comme marginalisés par rapport à la métropole britannique, sans autre forme de distinction. Cette opposition entre un centre impérial (Londres) et la périphérie des colonies est particulièrement développée dans les études postcoloniales de l'histoire indienne. Dans son étude de l'imaginaire indologique, Ronald Inden souligne ainsi le fait que les acteurs indiens (en général) seraient demeurés essentiellement en marge de leur propre histoire ${ }^{91}$. S'il est évident que les acteurs indiens n'étaient pas sur un pied d'égalité avec leurs collègues européens, il reste qu'Inden sous-estime sans doute leur rôle - y compris dans la production de savoir indologique - négligeant ainsi le caractère fécond des zones "marginales». Dans une acception rigoureusement géopolitique, les marges font ensuite référence aux zones limitrophes de l'Inde: en particulier le Pakistan, le Bangladesh, le Népal, le Bhoutan ou encore le Tibet, liés par une histoire commune mais séparés par des frontières nationales. Le traumatisme de la partition a laissé de profondes cicatrices dans les histoires respectives des nations qui en ont émergé. Ce simple constat a favorisé la réflexion sur les processus historiques de la constitution d'entités politiques, de centres et de périphéries, sur la construction patrimoniale d'une "communauté imaginée" (selon l'expression de Benedict Anderson ${ }^{92}$ ) s'accompagnant de la marginalisation d'histoires alternatives. A partir des cas pakistanais et bangladeshis, plusieurs travaux ${ }^{93}$ mettent en évidence le caractère construit

90. On peut mentionner dans ce sens les programmes de néoeurasianisme lancés au Kazakhstan et en Russie: M. Laruelle, La quête d'une identité impériale.

9I. R. B. Inden, Imagining India, p. 7-48.

92. B. Anderson, Imagined Communities.

93. G. Pandey, Remembering Partition et Ch. Haines, Nation, Territory, and Globalization in Pakistan, pour le Pakistan; R. Samaddara, The Marginal Nation, pour le Bangladesh; P. Titus, Marginality and Modernity, pour le Balouchistan. 
et artificiel de la nation et des frontières, en replaçant au centre de leur analyse les groupes sociaux eux-mêmes.

L'étude des marges sous un angle socio-anthropologique interroge le plus souvent les relations entre des groupes marginalisés et des groupes "majoritaires», dans le cadre défini d'un Etat-nation. Ainsi, s'appuyant sur les cas de pays d'Amérique latine, d'Afrique et d'Asie méridionale afin d'y analyser les marges socio-anthropologiques d'un Etat, Veena Das et Deborah Pool proposent de distinguer trois approches: une première portant sur la marge comme espace périphérique pour des groupes (souvent associés à une population "indigène" colonisée ou postcolonisée) jugés insuffisamment socialisés par rapport à une loi étatique; une deuxième s'intéressant à l'aspect de la marginalité par rapport au fonctionnement administratif et sécuritaire des Etats; une troisième, enfin, portant sur la marginalité par rapport à une norme biologique, représentée par la médecine et intégrée dans le corpus légal de l'Etat ${ }^{94}$.

Dans le monde russophone où les sciences humaines ont été traditionnellement et fondamentalement "élitistes ", l'analyse des "marges" sous divers angles sociaux, étatiques ou identitaires n’a que très récemment démarré, tandis que les publications, peu nombreuses, continuent à s'aligner sur les tendances des anthropologues occidentaux. Conditionnées par la restructuration brutale de la société consécutive à la dissolution de l'Union soviétique, par la formation des nouveaux Etats dans la périphérie ex-soviétique et par les crises économiques à répétition, ces études sont marquées par une perception essentiellement négative des «marges» et concentrent de ce fait leur attention sur la gestion des nouveaux groupes marginaux (chômeurs, migrants, petits entrepreneurs, populations appauvries, etc.) et sur le rapport direct que la problématique entretient avec le niveau de développement économique et les relations inter-ethniques ${ }^{95}$. Les publications occidentales sur les groupes marginaux de la société contemporaine russe - ciblant essentiellement les migrations - semblent être plus positives dans leur analyse.

Dans le contexte indien, la notion socio-anthropologique des marges a été en revanche largement explorée sous des angles divers, en se focalisant non pas sur l'Inde, le Pakistan ou le Bangladesh (notamment) pris

94. V. Das, D. Poole, Anthropology in the Margins, en particulier p. 9 sq.

95. Parmi les pionniers de cette problématique en Russie voir E. N. Starikov, I. N. Popova, Z. T. Golenkova, etc. 
en tant qu'espaces marginaux, mais sur les groupes marginalisés au sein de ces espaces. On peut songer ici aux travaux du collectif des Subaltern Studies qui ont remis en question le mythe d'une Inde socialement indivisible en relativisant l'importance des "élites indiennes" et revalorisant la figure du paysan indien et les classes "subalternes", en particulier dans la période qui précède l'indépendance ${ }^{96}$. Plus récemment, des études visent à retracer l'histoire des groupes marginalisés de la société indienne (femmes, réfugiés, pirates de l'Océan indien, résidents des établissements pénitentiaires, groupes tribaux [ädivāsī], etc.) en usant de méthodes d'histoire sociale d'abord développées dans le contexte européen ${ }^{97}$. Les approches récentes les plus originales sont peut-être celles qui insistent sur l'hétérogénéité de la société britannique de l'Inde coloniale et sur la présence de groupes subalternes au sein même de ce contexte social ${ }^{98}$.

Souvent associées au concept des frontières, les approches anthropologiques portent également sur les pratiques quotidiennes de communautés transnationales, parfois minoritaires ${ }^{99}$. Ces communautés sont présentées de manière de plus en plus positive comme des moteurs de transformation des identités nationales, foyers de regards critiques et productrices d'un imaginaire nouveau par rapport aux cultures locales et globales et, finalement, foyers - par anticipation - de la mondialisation ${ }^{100}$. De même, la question des présences féminines - qui font exception dans le milieu essentiellement masculin des orientalistes ou des élites savantes ${ }^{101}$ - représente une thématique majeure des marges anthropologiques russo-indiennes. Bien que plusieurs travaux s'inscrivant dans la perspective des études postcoloniales aient investigué ce

96. Voir, p. ex., le recueil classique de R. Guha, G. Ch. Spivak, Selected Subaltern Studies, ainsi que les travaux des autres membres du collectif, comme Samir Amin ou David Arnold.

97. Pour plusieurs exemples voir S. Agha, E. Kolsky, Fringes of Empire.

98. H. Fischer-Tiné, Low and Licentious Europeans.

99. P. ex., Ch. R. Abrar, On the Margin.

Ioo. Pour plusieurs exemples dans le contexte asiatique, voir les projets présentés dans le cadre du réseau de recherche <http://asianborderlands.net> (consulté le 01.02.2013).

IOI. Les orientalistes au sens "classique» ont été d'abord et avant tout des hommes localisés dans les "centres", travaillant sur des textes, données, objets émanant eux aussi de milieux masculins. 
sujet du Pamir à l'Inde à travers l'Asie centrale russe ${ }^{102}$, il s'agit là d'un champ particulièrement riche pour de futures recherches.

Repensées par les historiens de l'art, les "marges» se placent, entre autres, au centre des négociations nécessaires à la construction des empires: des collectionneurs originaires d'Etats impériaux, représentants du pouvoir impérial mais engagés dans une voie particulière, originale, révélant la fascination que peut exercer une culture dominée, relèvent des "marges" à travers une activité qui entraîne des inclusions interculturelles ${ }^{103}$.

Indépendamment de l'ancrage disciplinaire et des angles d'analyse choisis, les approches d'aujourd'hui soulignent toutes le rôle créatif joué par les «marges» et leurs composantes "fluides» et non statiques, ainsi que leur force dans la genèse de transformations. Ce changement notable est à souligner de manière explicite: initialement conçues comme essentiellement "passives» et guidées par des métropoles éloignées incarnant le centre (et l'Etat), lui-même défini en tant que producteur des discours de pouvoir et détenteur de la "vérité», les "marges» sont désormais reconnues comme un creuset propre d'invention et de créativité et deviennent finalement - en raison de ce potentiel - centrales. Vues comme un lieu métamorphique de transition et de différence, comme un espace de contacts, d'échanges, de chevauchements et d'hybridations où tout peut être modifié lors d'un passage/transfert, les «marges" sont désormais «réhabilitées» ${ }^{104}$. Elles sont souvent associées au changement de direction d'un regard - critique - qui peut même renverser le système traditionnel de distribution des centres-périphéries ${ }^{105}$. L'exemple des réflexions de Jorge Luis Borges (1899-1986) à propos de l'«Orient islamique", si différentes de celles des orientalistes occidentaux et

IO2. Par rapport à l'espace russophone voir K. Hokanson, "Russian Women Travellers in Central Asia and India». Pour l'Inde, on peut songer à G. Ch. Spivak, "Can the Subaltern Speak?», ou K. Teltscher, India Inscribed (qui évoque dans une étude au demeurant relativement "classique», la place des femmes de Jahangir dans les interactions avec les Européens et propose une relecture de la satī).

I03. M. Jasanoff, Edge of Empire.

I04. Les réflexions d'H. Bhabha sur le "third space» peuvent parfaitement s'appliquer dans ce cadre (H. Bhabha, The Location of Culture). De même, cette réflexion sur la fécondité des marges peut concerner les théories de la traduction: voir, p. ex., l'essai de G. Shree, "Writing Is Translating Is Writing Is Translating Is », qui insiste sur le rôle créatif de l'espace situé en marge d'une langue particulière.

I05. F. de Toro, «From Where to Speak?». 
conscientes du caractère artificiel des représentations construites, semble dans ce sens extrêmement parlant ${ }^{106}$.

\subsection{Les orientalismes des marges}

La configuration inhabituelle choisie pour cet ouvrage permet d'analyser la notion de "marges" à deux niveaux. D'abord, de manière classique et sous un angle global, comment une aire géographique, un groupe social, un discours, une langue deviennent-ils marginaux par rapport à d'autres, définis comme "centraux»? En quoi la politique et le comportement des acteurs de l'élite savante ont-ils contribué à accentuer ce processus, que ces acteurs soient locaux ou de la métropole, nombreux ou non, confortés dans une bonne position sociale ou mal subventionnés?

D'autre part, et de manière plus spécifique par rapport à la construction des connaissances relatives aux espaces non européens dans le monde contemporain, il s'agit de comprendre le mécanisme de création des zones marginales des études postcoloniales. De quelle manière les études sur l'histoire de l'orientalisme ont-elles privilégié certaines zones géographiques, des discours ou des acteurs particuliers en reléguant les autres dans une «zone grise» ou "zone d'oubli»? Comment s'est constituée la surface épistémologique hétérogène et polarisée entre "centre» et "périphéries"? Pourquoi certaines théories sur les rapports entre savoir et pouvoir ont-elles été rejetées, omises ou acceptées partiellement et sous condition, dans les traditions scientifiques des différents pays, entre autres de l'espace postsoviétique souvent considéré comme relevant des "marges»? Quel(s) rôle(s) les délimitations disciplinaires (notamment celles des Area Studies) ont-elles joué dans ce processus?

En orientant les réflexions dans cette direction, il convient de nuancer l'idée selon laquelle les traditions savantes des «marges» sont en "zone mal connue»: un bref aperçu de la bibliographie grandissante sur les orientalismes russe et indien (supra) montre, au contraire, une floraison de nouvelles approches, même si ces deux cas n'entrent pas dans les catégories pré-définies par Saïd. Il serait plus judicieux de dire que l'épithète "marginal» se rapporte au fait que les orientalismes russe et indien sont longtemps restés hors champ et sont de ce fait peu visibles, dans une zone en quelque sorte "grise» ou «oubliée».

Io6. Ibid.; I. Almond, «Borges the Post-Orientalist». 
Parce qu'ils ne sont pas conciliables avec une conception binaire des relations coloniales, ces «orientalismes» n'ont pas reçu immédiatement autant d'attention que d'autres formes qui sont devenues plus «classiques" dans la communauté scientifique. Marquée par des idées reçues, comme celles qui se réfèrent à la prétendue âme eurasiatique russe ou à l'absence d'histoire écrite en Inde, leur étude s'est souvent heurtée à des prises de position politiques, à un poids idéologique, ainsi qu'à des exigences particulières dans la mise en place de l'outillage linguistique nécessaire aux chercheurs en raison du caractère "hybride» de ces lieux.

Par rapport à ces différents aspects, notre approche n'envisage donc pas de se limiter exclusivement au phénomène des "marges" par contraste avec les normes étatiques, ni aux expressions géographiques (zones frontalières) ou sociales (groupes sociaux et interactions des individus avec les empires), mais souhaite étendre la problématique à l'espace extra- et inter-national en la plaçant surtout au niveau épistémologique. Dans ce sens, une place importante de l'analyse est accordée aux mécanismes de récupération des savoirs orientalistes par les acteurs locaux qui ont auparavant été considérés comme étant à la marge du processus de constitution des savoirs, alors qu'ils ont en réalité su contester les prétentions européennes à la supériorité.

Il s'agit donc de sortir d'une vision statique de la marginalité pour mettre à jour les processus dynamiques qui émergent de ces zones interstitielles. Cette approche sera d'autant plus fructueuse qu'elle sera entreprise dans une perspective comparative.

\subsection{Comparer les «marges»}

Les réflexions engagées sous un angle comparatif par les chercheurs (du monde occidental essentiellement) découlent de plusieurs phénomènes comme les bouleversements des situations géopolitiques et économiques dans l'espace eurasiatique dans le sens le plus large ou l'évolution de la recherche marquée par la réhabilitation de l'intérêt pour les études sur les empires et le colonialisme dans le cadre du système-monde, la «révolution des archives» dans l'espace postsoviétique et l'augmentation des échanges scientifiques. 
$\mathrm{Au}$ sein de l'histoire russo-soviétique ${ }^{107}$, les chercheurs commencent par revisiter la tradition déjà ancienne qui a conduit les "occidentalistes" (zapadniki), slavophiles, panslavistes et "orientalistes" (vostočniki) ${ }^{108}$ à dresser des parallèles avec les empires "classiques" européens comme ceux de la France ou de l'Angleterre, définis comme "maritimes" et "modernes». Tout en restant centrée sur l'«européanisation» et la "modernisation", cette approche - qui garde encore son potentiel heuristique - a failli éclipser toute autre possibilité de comparaison. Toutefois, avec l'enracinement du concept des empires et l'abandon progressif des modes de pensées basés sur l'isolationnisme et l'exception culturelle, les spécialistes abordent également des analyses comparatives avec divers pays de l'Europe centrale, comme l'Allemagne et la Pologne ${ }^{109}$. Puis, suivant la logique de ces démarches, la Russie/Union soviétique finit par entrer dans la catégorie des empires "non classiques", "prémodernes» et "continentaux" à côté non seulement de l'empire austro-hongrois des Habsbourg, mais également de celui des Ottomans, ce qui déplace ainsi les analyses en dehors d'un cadre strictement européen.

Plus difficile, mais très prometteuse, cette dernière voie permet finalement d'accorder la priorité à la comparaison des marges qui se forment aussi bien par rapport aux centres des empires ou sur la lisière des continents phares (essentiellement l'Europe), qu'en dehors des théories historiques dominantes qui, sans surprise, sont également occidentales. En plaçant résolument l'histoire russo-soviétique dans une perspective comparative, ces nouvelles tendances proposent des combinaisons novatrices. Recoupant parfois plusieurs marges ${ }^{110}$ et sans épuiser

I07. Pour les publications fondamentales qui ont prédéfini ce type d'approche de l'histoire russo-soviétique, voir K. Barkey, M. von Hagen, After Empire; D. Lieven, Empire; A. Miller, Rossijskaja imperija v sravnitel'noj perspektive; A. Miller, A. J. Rieber, Imperial Rule; J. Millward, Eurasian crossroads; A. L. Stoler, "Considerations on Imperial Comparisons".

I08. Sur ces courants scientifiques et idéologiques voir, p. ex., M. Bassin, Imperial Visions.

I09. Voir le numéro spécial de Kritika, 2009 (M. David-Fox, P. Holquist, A. M. Martin, Fascination and Enmity), consacré aux interactions entre la Russie et l'Allemagne; U. von Hirschhausen, J. Leonhard, Comparing Empires. Sur les difficultés de telles comparaisons: Sub Altera Specie, 2008.

IIO. B. Eschment, H. Harder, Looking at the Coloniser (comparaisons de l'Asie centrale avec l'Inde [A. Khalid], avec la Turquie ottomane [V. Adam] et avec l'Iran [L. Newby]); A. L. Edgar, «Bolshevism, Patriarchy, and the Nation»; A. Khalid, «Backwardness and 
complètement les possibilités heuristiques de cette approche, les publications pionnières abordent les interactions entre le monde russe-soviétique-postsoviétique et la Turquie ottomane ${ }^{111}$, l'Iran ${ }^{112}$, le Xinjiang ${ }^{113}$ et, même, le Moyen-Orient ${ }^{114}$, l'Algérie ${ }^{115}$ et l'Afrique subsaharienne ${ }^{116}$.

A l'égard de l'Inde, la démarche comparative permet d'abord de mettre à jour des processus relatifs à la construction du monde moderne et à ses spécificités sur une échelle globale. L'Inde apparaît alors comme un rouage important d'un «système-monde» se déployant à partir de la zone de l'Océan indien, et ce dès l'époque prémoderne ${ }^{117}$. Plus spécifiquement, d'importants travaux ont récemment cherché à comparer les processus de "vernacularisation» en Europe et en Inde ${ }^{118}$, à intégrer les événements non européens (et en particulier de l'Asie du Sud) comme des facteurs majeurs de la constitution du monde moderne globalisé

the Quest for Civilization»; M. Aust, R. Vul'pius, A. Miller, Imperium inter Pares; T. Uyama, Asiatic Russia; D. Motadel, "Islam and the European Empires». L'analyse de la cristallisation de la conscience nationale en Asie centrale est particulièrement intéressante dans cette optique, car les intellectuels turcophones de l'empire russe se sont largement inspirés des pratiques ayant lieu en Iran, en Turquie et en Europe.

III. La comparaison de la Russie avec la Turquie au point de vue de l'orientalisme/occidentalisme semble extrêmement prometteuse dans la mesure où ce dernier pays est profondément marqué par des préjugés tels que sa "singularité culturelle», sa fermeture aux avancées théoriques postcoloniales, son rôle ambivalent d' «objet»-«sujet» et sa position entre l'"Orient» et l'"Occident». Voir le numéro spécial de Kritika, Models on the Margins. Russia and the Ottoman Empire, issu du colloque tenu à SaintPétersbourg en 2009 sur «Models on the Margins: Russia, the Ottoman Empire, and Spain» (l'Espagne n'a pas été retenue dans le recueil final); ainsi que M. A. Reynolds, Shattering Empires. Voir également le colloque tenu à l'Institut d'études politiques de Rennes "L'orientalisme désorienté? La Turquie contemporaine au miroir des approches postcolonialistes" en janvier 2010: <http://www.crape.univ-rennes1.fr/ documents/2010/Orientalisme_affiche.pdf> (consulté le 02.02.2013).

II2. E. Andreeva, Russia and Iran in the "Great Game"; S. Cronin, Iranian-Russian Encounters.

II3. L. Newby, The Empire and the Khanate.

II4. S. N. Cummings, R. Hinnebusch, Sovereignty after Empire.

II5. V. O. Bobrovnikov, "Russkij Kavkaz i frantsuzskij Alžir».

II6. D. Chioni Moore, "Is the Post- in Postcolonial the Post- in Post-Soviet?", p. $114 s q$.

II7. L'un des travaux pionniers est celui braudélien de K. N. Chaudhuri, Trade and Civilisation in the Indian Ocean, suivi par de nombreux autres, comme J. L. AbuLughod, Before European Hegemony, et plus récemment S. H. Smith, Reinterpreting Indian Ocean Worlds, ou Ph. Beaujard, Les mondes de l'océan Indien. II8. Sh. Pollock, The Language of the Gods in the World of Men. 
et de ses "métropoles» ${ }^{119}$ ou à contraster les expériences de groupes marginaux dans le contexte géographique de l'Océan indien ${ }^{120}$. La comparaison d'institutions, par exemple religieuses, issues du monde indien avec des correspondants non européens est par ailleurs reconnue dans une fonction heuristique consistant à se distancier des grilles d'analyse "canoniques» occidentales ${ }^{121}$. Ces différents travaux ont contribué à défaire l'idée d'une Inde «incomparable», spécialement pour ce qui concerne l'époque ancienne.

Il reste cependant que le potentiel heuristique et critique du regard comparatif est loin d'être épuisé, en particulier pour ce qui concerne la période moderne et contemporaine. Dans ce sens, la comparaison entre la Russie/URSS et l'Inde, sous l'angle de la production des savoirs orientalistes, peut s'avérer fort fructueuse, même si elle n'a pour diverses raisons pas encore reçu toute l'attention qu'elle mérite. Par exemple, on attend toujours des initiatives dans la comparaison entre les processus de décolonisation de l'Asie centrale ou du Caucase d'une part, et ceux de l'Inde d'autre part.

Figé essentiellement sur les espaces du Proche- ou du Moyen-Orient et du Maghreb, le terrain de prédilection de l'orientalisme a été géographiquement délimité par les premières analyses de Saïd. En même temps, la direction des analyses s'est fixée de manière unilatérale le long d'un axe unique métropole-colonie. Tributaires de cette approche et des critiques qui s'en sont suivies, les cas de la Russie et de l'Inde ont été souvent décrits selon des logiques distinctes: d'un côté, la Russie s'est vue présentée à la lisière de l'Europe comme une puissance hybride eurasiatique «trop particulière» et «incompatible» avec la critique postcoloniale, d'un autre côté, l'Inde a obtenu le statut de colonie la plus riche de l'Empire britannique. De ce fait, elle est devenue emblématique du système colonial et est apparue comme tout à fait similaire aux autres colonies "classiques» tant au Moyen-Orient qu'en Afrique. Ainsi associés aux deux pôles opposés du système colonial, ces deux cas ont d'emblée été déclarés comme "incomparables", les rares tentatives de mise en parallèle étant reléguées dans les marges de la critique postcoloniale.

II9. Ch. A. Bayly, The Birth of the Modern World.

I20. C. Anderson, Subaltern Lives.

I2I. Ainsi Ph. Bornet, Rites et pratiques de l'hospitalité; B. Holdrege, «The Politics of Comparison". 
Par ailleurs, les problèmes méthodologiques ont été accentués par le décalage chronologique des deux exemples. Premièrement, il est difficile de dresser des parallèles exacts entre le demi-siècle de présence russe impériale au Turkestan (des environs de 1860 à 1917, l'époque soviétique de 1917 à 1991 sortant de ce cadre de manière discutable) et l'histoire des Indes britanniques qui combine la bien plus longue gouvernance de l'East India Company (dès le XVII siècle et de manière plus appuyée à peu près depuis 1757) et du Raj britannique (de 1858 à 1947). Enfin, il ne semble pas possible de mettre à égalité les expériences postcoloniales de l'Inde qui a eu accès à l'indépendance en 1947 et celles des républiques soviétiques qui n'ont obtenu qu'en 1991 le statut d'Etats indépendants ${ }^{122}$.

Or, malgré le poids de la tradition et des obstacles de nature diverse, cette situation pourrait s'inverser, si elle n’a pas déjà commencé à le faire. A part le fait que les débats sur l'orientalisme russe sont déjà accueillis dans la recherche indienne ${ }^{123}$, rappelons que les premières tentatives d'approches comparées ont démarré tout d'abord dans le domaine de l'histoire préimpériale et impériale avant de passer dans le domaine des politistes. Ces derniers font cependant aujourd'hui appel à l'image d'un nouveau Great Game dont l'analyse continue de marginaliser les Centrasiatiques au profit des grandes puissances traditionnelles avoisinantes ${ }^{124}$. En commençant par la mise en perspective des relations commerciales entre l'Asie centrale et l'Inde ${ }^{125}$, les chercheurs sont passés à la comparaison du pouvoir colonial russe avec les pratiques du Raj

I22. Sur les difficultés d'établir les dates exactes de la présence russe au Turkestan et la durée du pouvoir soviétique dans cette même région voir S. Gorshenina, S. Abashin, Le Turkestan russe, p. 9 sq. Ce problème de décalage chronologique a également été relevé par A. S. Morrison, Russian Rule in Samarkand, 1868-1910, p. 3, et S. N. Abašin, «Zavoevanie Srednej Azii».

I23. Tout en manifestant un certain intérêt pour la problématique de l'orientalisme russo-soviétique, les russisants indiens restent plutôt spectateurs des discussions postsoviétiques, sans vouloir tenter d'appliquer au cas russe l'expérience des approches formulées par les études postcoloniales indiennes.

I24. Sur les premières tentatives fructueuses d'introduire l'Inde comme acteur à part entière dans l'analyse de la situation géopolitique en Asie centrale voir M. Laruelle et al., China and India in Central Asia; M. Laruelle, S. Peyrouse, L'Asie centrale à l'aune de la mondialisation et Mapping Central Asia. Egalement E. Kavalski, India and Central Asia.

I25. Th. Zarcone, Inde-Asie centrale; S. Levi, India and Central Asia. 
britannique où les orientalismes russe et indien sont compris comme des techniques de pouvoir impérial et sont explicitement analysés à travers la grille des études postcoloniales ${ }^{126}$.

Ainsi, les "orientalist packages" qui semblent si différents pour la Russie et l'Inde peuvent - et doivent - être comparés à la condition que le cas russe soit défait de tout caractère "exceptionnel", et que, symétriquement, le cas indien ne soit pas considéré comme aussi «classique» que ce qui est souvent affirmé.

Ces entreprises comparatives, à mener dans un contexte de collaboration académique entre des spécialistes des différents domaines concernés, pourront s'articuler autour de deux axes méthodologiques.

Premièrement, de la manière la plus traditionnelle, la comparaison de situations liées sur le plan historique permet de mettre en lumière des processus d'interaction entre deux contextes ou «mondes» souvent conçus comme cloisonnés par les historiographies "traditionnelles" ou nationales. Dans le cas qui nous occupe, outre les sujets déjà amorcés sur les voyageurs et les emprunts artistiques et littéraires, on peut penser au courant de la théosophie, à la réception russe d'auteurs comme Tagore, à l'histoire du marxisme indien ou à l'histoire de zones «tampons» (Great Game par exemple).

Deuxièmement, il convient de souligner que le travail comparatif ne présuppose pas nécessairement des contacts historiques directs ou généalogiques. L'essentiel consistera alors à ne pas s'attacher seulement à quelques moments présentant une certaine similarité, mais au contraire à souligner les différences d'un contexte à l'autre, avec une problématique construite en amont - à savoir, pour le sujet qui nous occupe, celle de la construction des savoirs dans une situation coloniale et leur impact aux niveaux politique et social ${ }^{127}$. De la sorte et de manière particulièrement

I26. Sans toujours réussir à dépasser les pièges de l'analyse comparative, où les exemples indiens, réduits à un rôle d'illustrations, sont épisodiquement convoqués afin de mettre en lumière des processus similaires qui se sont déroulés sur sol russe, Alexander Morrison, le chercheur le plus appliqué actuellement dans cette problématique, tente surtout de comprendre si le Turkestan russe joue le même rôle pour la Russie que l'Inde pour l'Angleterre: A. S. Morrison, Russian Rule in Samarkand, 18681910 et " "Applied Orientalism” in British India and Tsarist Turkestan».

I27. Sur quelques principes fondamentaux de la "comparaison différentielle», voir U. Heidmann, "Comparatisme et analyse de discours» et "La comparaison différentielle comme approche littéraire». Sur les difficultés de cette approche comparative, voir 
significative, la comparaison entre diverses "marges" hors de tous contacts pourra faire ressortir l'existence de plusieurs niveaux de pratiques et de langages dont la nature change selon les destinataires (au centre, dans le même espace périphérique, ou dans les autres "marges») et selon la localisation géographique des émetteurs.

Ces réflexions pourront s'aligner sur les nouvelles approches qui prennent forme dans les exemples relatifs aux autres aires culturelles, à l'interface de plusieurs méthodes comme les transferts culturels (Michel Espagne, Michael Werner), l'histoire croisée, l'histoire «à parts égales", l'histoire connectée (Serge Gruzinski, Romain Bertrand, Sanjay Subrahmanyam), l'histoire transnationale, ou la micro-histoire (Carlo Ginzburg, Jacques Revel), les phénomènes d'hybridation (Homi Bhabha) et de métissage (Alexis Nouss), les shared/entangled histories (Sidney Mintz), etc.

Dans le contexte spécifique de la Russie et de l'Inde, on pourra ainsi mieux comprendre les mécanismes de l'impérialisme russe par comparaison avec le cas indien, bien documenté et largement étudié. En corrélant l'histoire russo-soviétique à l'expérience coloniale des autres puissances ${ }^{128}$ plusieurs démarches paraissent particulièrement prometteuses, comme l'identification des parallélismes entre les histoires des empires au moment du Great Game, ou la comparaison des diverses manières d'étudier les espaces orientaux en dehors du cadre de l'opposition entre la "progressiste et démocratique» Russie et

les remarques importantes de M. Espagne, «Sur les limites du comparatisme en histoire culturelle».

I28. Dans le monde russe postsoviétique, la Russie est toujours vue comme une puissance profondément non coloniale en raison d'une interprétation forgée depuis les années 1930. Voir, p. ex., dans un manuel récent d'introduction aux études orientales, l'affirmation selon laquelle l'orientalisme russo-soviétique «n'a jamais été instrument de la soumission coloniale, mais, au contraire, étudie en parallèle avec l'Asie étrangère ses propres espaces asiatiques»: E. I. Zelenev, V. B. Kasevič, Vvedenie v vostokovedenie, p. 12 sq., 25. Pour une même observation sur la perception extrêmement négative qu'ont les chercheurs russes de l'époque postsoviétique à l'égard des interprétations relatives au caractère "colonialiste» du passé russe, voir A. S. Morrison, Russian Rule in Samarkand, 1868-1910, p. 8. Soulignons cependant que la perception du passé tsariste n'est pas la même dans les ex-républiques soviétiques qui ont chacune élaboré une propre version distincte. Sur diverses approches forgées par les chercheurs russes, soviétiques et postsoviétiques à l'égard du colonialisme russe voir S. Gorshenina, «La marginalité du Turkestan colonial russe est-elle une fatalité, ou l'Asie centrale postsoviétique entrera-telle dans le champ des Post-Studies?", p. 19-47. 
l'«impérialiste» Angleterre, voire le rôle des élites nationales dans la construction des connaissances et dans l'écriture des histoires nationales. Inversement, la position prétendument particulière et ambivalente de la Russie à l'égard de l'orientalisme invite à diriger le regard vers les acteurs «locaux» de l'orientalisme en Inde. En s'éloignant d'une simple critique des orientalistes occidentaux, la comparaison avec différentes situations analogues dans le monde russe pourra s'avérer éclairante pour retracer les histoires complexes de courants d'idées et de pratiques savantes en Inde. Des sujets comme ceux de l'écriture d'histoires de l'Inde, des politiques linguistiques, ou encore du développement d'idéologies s'appuyant sur une opposition entre Asie et Europe pourront ainsi bénéficier d'une perspective comparée.

Pour ces différentes raisons, nous avons renoncé à organiser les contributions de cet ouvrage par aire culturelle (Inde, Russie), de sorte à sortir d'une conception réifiée de ces domaines et pour mettre en évidence les connexions - que ce soit sur un plan historique ou analytique - qui relient les différents cas étudiés.

Ainsi entendue, la comparaison placée au carrefour de la pluridisciplinarité et agissant comme antidote à l'«hyperspécialisation » du monde académique contemporain permet d'éclairer des faits déjà étudiés sous un angle nouveau et de briser toute prétention à l'exemplarité. Prise dans la dialectique de l'inclusion et de l'exclusion, la notion d'" orientalisme des marges" semble porteuse d'une tension intrinsèque féconde. Elle implique en effet de reconsidérer le modèle binaire associé à la notion d'orientalisme depuis Saïd. Elle force à considérer les processus de constitution des savoirs en situation coloniale sous un angle dynamique et dirige l'attention sur les processus ou les acteurs issus de contextes «marginalisés ", ce qui permet de renouveler la problématique, déjà devenue classique, de l'orientalisme.

Philippe BORnet

Université de Lausanne

Svetlana Gorshenina

FNS, Université de Lausanne et University of Manchester 


\section{BIBLIOGRAPHIE}

Aваšı̀, Sergej Nikolaevič, «Razmyšlenija o "Central'noj Azii v sostave Rossijskoj imperii" ", Ab Imperio, 4 (2008), p. 456-471.

—, «Nacii i postkolonializm v Central'noj Azii dvadcat' let spustja: pereosmyslivaja kategorii analiza/praktiki», Ab Imperio, 3 (2011), p. 193-210.

—, "Zavoevanie Srednej Azii : imperskij i nacional'nyj narrativy", communication au colloque Mir imperij: učenye, znanija $i$ «iskusstvo upravlenija" periferiej v kolonial'nyh $i$ kontinental'nyh imperijah, 12-14 novembre 2012, Moscou.

Abašin, Sergej N., Arapov, Dimitri Ju., Bekmahanova, N. E. (éds), Central'naja Azija v sostave Rossijskoj Imperii, Moskva, Novoe literaturnoe obozrenie, 2008.

Abdel-Malek, Anouar, "L'orientalisme en crise», Diogène, 44 (1963), p. $109-142$.

Abrar, Chowdhury Rafiqul (ed.), On the Margin: Refugees, Migrants and Minorities, Dhaka, Refugee and Migratory Movements Research Unit, 2000.

Abu-Lughod, Janet L., Before European Hegemony: The World System A.D. 1250-1350, New York/Oxford, Oxford University Press, 1989.

Adams, L., "Can We Apply Postcolonial Theory to Central Eurasia?», Central Eurasian Studies Review, 7/1 (2008), p. 2-7.

Agra, Sameetah, Kolsky, Elizabeth, Fringes of Empire: Peoples, Places, and Spaces in Colonial India, New York, Oxford University Press, 2009.

Ahmad, Aijaz, "Orientalism and After: Ambivalence and Metropolitan Location in the Work of Edward Said", in In Theory: Classes, Nations, Literatures, London, Verso, 1992, p. 159-220.

Alatorre, Sophie, "An Approach to Renaissance Margins: Some Representations of the Labyrinth on the Elizabethan Stage", E-rea: Revue électronique d'études sur le monde anglophone, 6/1 (2008), mis en ligne le 15 octobre 2008, consulté le 10 janvier 2013: <http://erea.revues.org/95>. 
Almond, Ian, "Borges the Post-Orientalist: Images of Islam from the Edge of the West", MFS Modern Fiction Studies, 50/2 (2004), p. 435-459.

Almond, Philip, The British Discovery of Buddhism, Cambridge, Cambridge University Press, 1988.

Ames, Eric, Klotz, Marcia, Wildenthal, Lora (eds), Germany's Colonial Pasts, Lincoln, University of Nebraska Press, 2005.

Anderson, Benedict, Imagined Communities: Reflections on the Origin and Spread of Nationalism, London, Verso, 1983.

Anderson, Clare, Subaltern Lives: Biographies of Colonialism in the Indian Ocean World, 1790-1920, Cambridge, Cambridge University Press, 2012.

Andreev, Aleksandr, Hram Buddy v Severnoj stolice, Sankt-Peterburg, Nartang, 2004.

Andreeva, Ekaterina, Russia and Iran in the "Great Game": Travelogues and Orientalism, London, Routledge, 2008.

Antonova, Koka Aleksandrovna, Gol’dberg, Nikolaj Maksimovič, LaVrencova, T. D. (éds), Russko-indijskie otnošenija v XVII v. Sbornik dokumentov, Moskva, Izdatel'stvo vostočnoj literatury, 1958.

App, Urs, "How Amida Got into the Upanishads: An Orientalist's Nightmare", in Essays on East Asian Religion and Culture. Festschrift in Honour of Nishiwaki Tsuneki on the Occasion of his 65th Birthday, ed. by C. Wittern, S. Lishan, Kyoto, 2007, p. 11-33.

-, The Birth of Orientalism, Philadelphia, University of Pennsylvania Press, 2012.

Arnold, David, Colonizing the Body: State Medicine and Epidemic Disease in Nineteenth-Century India, Berkeley/Los Angeles, University of California Press, 1993.

-, Science, Technology and Medicine in Colonial India, Cambridge, Cambridge University Press, 2000.

Aust, Martin, Vul'pius, Rikarda [Ricarda Vulpius], Miller, Aleksej (éds), Imperium inter Pares: Rol' transferov v istorii Rossijskoj imperii (1700-1917), Moskva, Novoe literaturnoe obozrenie, 2010.

BabaDŽAnov, B. M., Kokandskoe hanstvo: vlast', politika, religija, Tokyo/ Tachkent, 2010. 
Baberovskij, Ïorg [Jörg Baberowski], Vrag est'vezde: Stalinism na Kavkaze, Moskva, Rosspen, 2010.

Balagangadhara, S. N., "The Heathen in his Blindness... ": Asia, the West and the Dynamic of Religion, Leiden/New York, Brill, 1994.

Balandier, G., "La situation coloniale: approche théorique», Cahiers internationaux de sociologie, 110/1 (1951), p. 9-29.

Barkey, Karen, von Hagen, Mark (eds), After Empire: Multiethnic Societies and Nation-Building. The Soviet Union and the Russian, Ottoman, and Habsburg Empires, Boulder, CO, Westview, 1997.

Bartol'D, V. V., Istorija izučenija Vostoka v Evrope i v Rossii, Moskva, Nauka, 1977 (traduction française: V. V. BARTHOLD, La découverte de l'Asie: Histoire de l'orientalisme en Europe et en Russie, traduit par B. Nikitine, Paris, Payot, 1947).

BAssin, Mark, Imperial Visions. Nationalist Imagination and Geographical Expansion in the Russian Far East, 1840-1865, Cambridge, Cambridge Univ. Press, 1999 (Cambridge Studies in Historical Geography 29).

—, "Eurasianism "Classical" and "Neo": The Lines of Continuity", Slavic Eurasian Studies, 17 (2008), p. 279-294: <http://src-h.slav. hokudai.ac.jp/coe21/publish/no17_ses/14bassin.pdf> (consulté le 03.01.2013).

BAYART, Jean-François, Les études postcoloniales: Un carnaval académique, Paris, Karthala, 2010 (Disputatio).

BAyly, Christopher A., Indian Society and the Making of the British Empire, Cambridge, Cambridge University Press, 1989.

—, The Birth of the Modern World: 1780-1914: Global Connections and Comparisons, Malden, Blackwell, 2004.

BAYly, Susan, "Imagining "Greater India”: French and Indian Visions of Colonialism in the Indic Mode", Modern Asian Studies, 38/3 (2004), p. 703-744.

Bazijanc, Ašot P., Bertel's, D. E., Aziatskij muzej-Leningradskoe otdelenie Instituta vostkovedenija AN SSSR, Moskva, Nauka, 1972.

Beaujard, Philippe, Les mondes de l'océan Indien, Paris, A. Colin, 2012. Belov, E. L., Svjateckaja, O. I., Šaumjan, T. L., Rossija i Tibet: sbornik russkih arhivnyh dokumentov 1900-1914, Moskva, Vostočnaja literatura, 2005.

Ben-Ghiat, Ruth, Fuller, Mia (eds), Italian Colonialism, London, Palgrave Macmillan, 2005. 
Bhabha, Homi, The Location of Culture, London, Routledge, 1994.

Birgerson, Susanne Michele, After the Breakup of a Multi-Ethnic Empire: Russia, Successor States, and Eurasian Security, Westport, Praeger, 2002.

Birman, D. A., Kotovsкij, G. G., Sosina, N. N., Bibliografija Indii: dorevoljucionnaja i sovetskaja literatura na russkom jazyke i jazykah narodov SSSR, original'naja i perevodnaja, Moskva, Nauka, 1976. Bobrovnikov, Vladimir O., "Počemu my marginaly? Zametki na poljah russkogo perevoda "Orientalizma” Edvarda Saida", $A b$ Imperio, 2 (2008), p. 325-344.

—, «Russkij Kavkaz i frantsuzskij Alžir: Slučajnoe shodstvo ili obmen opytom kolonial'nogo stroitel'stva?", in Imperium inter Pares: Rol' transferov $v$ istorii Rossijskoj imperii (1700-1917), ed. by M. Aust, R. Vul'pius, A. Miller, Moskva, Novoe literaturnoe obozrenie, 2010, p. 182-209.

Boeck, Brian J., Imperial Boundaries: Cossack Community and EmpireBuilding in the Age of Peter the Great, Cambridge, Cambridge University Press, 2009.

Bongard-Levin, G. M., Vigasin, A. A., The Image of India: The Study of Ancient Indian Civilisation in the USSR, transl. from the Russian by Peter Greenwood, Moscow, Progress Publishers, 1984. Bornet, Philippe, Rites et pratiques de l'hospitalité: mondes juifs et indiens anciens, Stuttgart, Steiner, 2010.

Breckenridge, Carol A., van der Veer, Peter (eds), Orientalism and the Postcolonial Predicament: Perspectives on South Asia, Philadelphia, University of Pennsylvania Press, 1993.

Breyfogle, Nicholas B., Schrader, Abby, Sunderland, Willard, Peopling the Russian Periphery: Borderland Colonization in Eurasian history, London/New York, Routledge, 2007.

Burbank, Jane, Cooper, Frederick, Empires in World History: Power and the Politics of Difference, Princeton, Princeton University Press, 2010.

Carlson, Maria, "No Religion Higher than Truth": A History of the Theosophical Movement in Russia, 1875-1922, Princeton, Princeton University Press, 1993.

Chanrabarty, Dipesh, Provincializing Europe, Princeton, Princeton University Press, 2000. 
Chatterjee, Kumkum (ed.), Europe Observed: Multiple Gazes in Early Modern Encounters, Lewisburg, Bucknell University Press, 2008.

Chatterjee, Partha, "Alternative Nations, Alternative Histories", in Making Alternative Histories: The Practice of Archaeology and History in Non-Western Settings, ed. by P. Schmidt, T. Patterson, Santa Fe, School of American Research, 1996, p. 229-253.

Chaudhuri, Kirti N., Trade and Civilisation in the Indian Ocean: An Economic History from the Rise of Islam to 1750, Cambridge, Cambridge University Press, 1985.

Chioni Moore, David, "Is the Post- in Postcolonial the Post- in PostSoviet? Toward a Global Postcolonial Critique», PMLA, 116/1 (January 2001), Special Topic: Globalizing Literary Studies, p. 111-128.

Choné, Aurélie, Rudolf Steiner, Carl Gustav Jung, Hermann Hesse: Passeurs entre Orient et Occident. Intégration et transformation des savoirs sur l'Orient dans l'espace germanophone (1890-1940), Strasbourg, Presses universitaires de Strasbourg, 2009.

Clarkson, Stephen, "Le modèle soviétique de développement pour l'Inde", Revue de l'Est, 3/3 (1972), p. 131-149.

—, "Non-Impact of Soviet Writing on Indian Thinking and Policy", Economic and Political Weekly, 15/8 (1973), p. 715-724.

Clifford, James, "Review of Edward Said's Orientalism", History and Theory. Studies in the Philosophy of History, 19 (1980), p. 204-223.

Conn, Bernard, "The Command of Language and the Language of Command", in Subaltern Studies: Writing on South Asian History and Society IV, ed. by R. Guha, Delhi, Oxford University Press, 1985, p. 276-329.

-, An Anthropologist among the Historians and Other Essays, Delhi/ Oxford, Oxford University Press, 1987.

—, "Cloth, Clothes, and Colonialism: India in the Nineteenth Century", in Colonialism and its Forms of Knowledge: The British in India, Princeton, Princeton University Press, 1996, p. 106-161.

Сомтет, Roger (éd.), Les Russes et l'Orient, Slavica Occitania, 8 (1999), Toulouse.

Cooper, Frederick, Stoler, Ann Laura, Tensions of Empire: Colonial Cultures in a Bourgeois World, Berkeley/Los Angeles, University of California Press, 1997. 
Cronin, Stephanie (ed.), Iranian-Russian Encounters. Empires and Revolutions Since 1800, Routledge, Iranian Studies, 2012.

Cummings, Sally N., Hinnebusch, Raymond (eds), Sovereignty after Empire: Comparing the Middle East and Central Asia, New York/ Edinburgh, Columbia University Press/Edinburgh University Press, 2011.

ČAč, Elena A., Orientalizm v obščestvennom i hudožestvennom soznanii Serebrjannogo veka, thèse kandidatskaja en histoire, SanktPeterburg, 2012.

Das, Veena, Poole, Deborah, Anthropology in the Margins, Santa Fe/ Oxford, School of American Research Press/James Currey Ltd, 2004.

David-Fox, Michael, Holquist, Peter, Martin, Alexander M.,

Orientalism and Empire in Russia, Bloomington, Slavica, 2006.

- (eds), "The Imperial Turn», special issue of Kritika, 7/4 (2006), p. 705-712.

- (eds), Fascination and Enmity: Russia and Germany as Entangled Histories, 1914-1945, Pittsburgh, University of Pittsburgh, 2012 (repr. special issue of Kritika, 10/3 [2009]).

Dirks, Nicholas, Castes of Mind: Colonialism and the Making of Modern India, Princeton, Princeton University Press, 2001.

Di Ruocco, Adele, The Buddhist World in Modern Russian Culture (1873-1919): Literature and Visual Arts, Ph. D. thesis, University of Southern California, 2011.

Dodson, Michael, Orientalism, Empire, and National Culture: India, 1770-1880, Basingstoke/New York, Palgrave Macmillan, 2007.

Dudden, Alexis, Japan's Colonization of Korea: Discourse and Power, Honolulu, University of Hawai'i Press, 2005.

Edgar, Adrienne Lynn, "Bolshevism, Patriarchy, and the Nation: The Soviet "Emancipation " of Muslim Women in Pan-Islamic Perspective", Slavic Review, 65/3 (2006), p. 252-272.

Erkinov, Aftandil, Praying for and against the Tsar: Prayers and Sermons in Russian Dominated Khiva and Tsarist Turkestan, Berlin, Klaus Schwarz Verlag, 2004 (Anor 16).

Eschment, Beate, Harder, Hans (eds), Looking at the Coloniser: CrossCultural Perceptions in Central Asia and the Caucasus, Bengal, and Related Areas, Würzburg, Ergon Verlag, 2004 (Mitteilungen zur Sozial- und Kulturgeschichte der islamischen Welt 14). 
Espagne, Michel, "Sur les limites du comparatisme en histoire culturelle», Genèses, 17 (1994), p. 112-121.

ETKInd, Alexander, Internal Colonization: Russia's Imperial Experience, Cambridge, Polity Press, 2011.

Etrind, Alexander, Uffelmann, Dirk, Kunulin, Ilia (éds), Tam, vnutri. Praktiki vnutrennej kolonizacii v kul'turnoj istorii Rossii, Moskva, Novoe literaturnoe obozrenie, 2012.

Ferrari, Aldo, La foresta e la steppa. Il mito dell'Eurasia nella cultura russa, Milano, Libri Scheiwiller, 2003.

Figes, Orlando, Natasha's Dance: A Cultural History of Russia, New York, Picador, 2002.

Fischer-Tiné, Harald, Low and Licentious Europeans: Race, Class and White Subalternity in Colonial India, Delhi, Orient Black Swan, 2009.

Fiskovec, E. V., Obraz Indii v russkoj literature, thèse kandidatskaja en littérature, Petrozavodsk, 2011.

Forum AI, "The Imperial Turn in Russian Studies: Ten Years Later (Sheila Fitzpatrick, USA; Paul Werth, USA; Mark von Hagen, USA; Malte Rolf, Germany; Svetlana Gorshenina, France; Jane Burbank, USA; Zaur Gasimov, Germany; Serhy Yekelchyk, Canada; Darius Staliunas, Lithuania)", Ab Imperio, 1 (2010), p. 64-88.

—, "Obsuždenie knigi Very Tolz, Russia's Own Orient: The Politics of Identity and Oriental Studies in the Late Imperial and Early Soviet Periods (Oxford, Oxford University Press, 2011), 203 p., avec Mikhail Dolbilov, "O vygode imet' 'svoj sobstvennyj Vostok'” [p. 374-384] ; Sergei Glebov, "Postcolonial Empire? Russian Orientologists and the Politics of Knowledge in Late Imperial Russia” [p. 385-392] ; Vladimir Bobrovnikov, "Novaja kniga Very Tol'c: kommentarij vostokoveda" [p. 393-402]; Vera Toltz, "otvet recenzentam" [p. 403-409]", Ab Imperio, 3 (2011), p. 374-409.

Friedrichsmeyer, Sara, Lennox, Sara, Zantop, Susanne (eds), The Imperialist Imagination: German Colonialism and its Legacy, Ann Arbor, University of Michigan Press, 1998.

Geraci, Robert P., Window on the East: National and Imperial Identities in Late Tsarist Russia, Ithaca, Cornell University Press, 2001. 
Geraci, Robert P., Khodarskovsky, Michael (eds), Of Religion and Empire: Missions, Conversion, and Tolerance in Tsarist Russia, Ithaca, Cornell University Press, 2001.

Gorshenina, Svetlana, «La marginalité du Turkestan colonial russe est-elle une fatalité, ou l'Asie centrale postsoviétique entrera-t-elle dans le champ des Post-Studies?», in Le Turkestan russe: une colonie comme les autres?, éd. par S. Gorshenina, S. Abashin, Cahiers d'Asie centrale, 17/18 (2009), p. 17-78.

-, Asie centrale: L'invention des frontières et l'héritage russo-soviétique, Paris, CNRS-Editions, 2012 (Espaces et milieux).

Goršenina [Gorshenina], Svetlana, Čuhovič [Chukhovich], Boris, "Srednjaja Azija kak fenomen čistogo orientalističeskogo èksperimenta (1860-1990-e gg.)", in Transoxiana. History and Culture, Tashkent, Institut Iskusstvoznanija Akademii hudožestv Uzbekistana/Institut Otkrytoe Obščestvo, 2004, p. 339-346: $<$ http://svetlana.gorshenina.free.fr/1_Experience_Orientaliste_ Transoxiana.pdf $>$.

Gorshenina, Svetlana, Abashin, Sergej (éds), Le Turkestan russe: une colonie comme les autres?, Cahiers d'Asie centrale 17/18, Paris, Complexe, 2009.

Gruen, Erich S., Rethinking the Other in Antiquity, Princeton/ Woodstock, Princeton University Press, 2011.

Guna, Ranajit, History at the Limit of World-History, New York, Columbia University Press, 2002.

Guha, Ranajit, Spivak, Gayatri Chakravorty (eds), Selected Subaltern Studies, New York/Oxford, Oxford University Press, 1988.

Haines, Chad, Nation, Territory, and Globalization in Pakistan: Traversing the Margins, London/New York, Routledge, 2012.

Haithcox, John Patrick, Communism and Nationalism in India. M. N. Roy and Comintern Policy 1920-1939, Princeton, Princeton University Press, 1971.

Halbfass, Wilhelm, India and Europe: An Essay in Understanding, New York, SUNY, 1988 [1981].

Halfin, N. A., Šastitko, P. M. (éds), Indija i Rossija, Moskva, Nauka, 1986.

Hay, Stephen N., Asian Ideas of East and West: Tagore and his Critics in Japan, China, and India, Cambridge, Harvard University Press, 1970. 
Heidmann, Ute, "Comparatisme et analyse de discours. La comparaison différentielle comme méthode», in Sciences du texte et analyse de discours. Enjeux d'une interdisciplinarité, éd. par J.-M. Adam, U. Heidmann, Genève, Slatkine, 2005, p. 99-118.

—, "La comparaison différentielle comme approche littéraire", in Nouveaux regards sur le texte littéraire, éd. par V. Jouve, Reims, Editions et Presses universitaires de Reims, 2013, p. 203-222.

Hirsch, Francine, Empire of Nations: Ethnographic Knowledge and the Making of the Soviet Union, Ithaca/London, Cornell University Press, 2005.

Hirschhausen, Ulrike von, Leonhard, Jörn (eds), Comparing Empires: Encounters and Transfers in the Long Nineteenth Century, Göttingen, Vandenhoeck \& Ruprecht, 2011.

Hokanson, Kaya, "Russian Women Travellers in Central Asia and India", Russian Review, 70/1 (2011), p. 1-19.

Holdrege, Barbara, "The Politics of Comparison: Connecting Cultures Outside of and in Spite of the West», International Journal of Hindu Studies, 14/2-3 (2010), p. 147-175.

Inden, Ronald B., Imagining India, Oxford/Cambridge (Mass), Blackwell, 1992.

Irschick, Eugène F., Dialogue and History: Constructing South India, 1795-1895, Berkeley, University of California Press, 1994.

IRwIN, Robert, For the Lust of Knowing: The Orientalists and their Enemies, London, Allen Lane, 2006.

Jasanoff, Maya, Edge of Empire: Lives, Culture, and Conquest in the East, 1750-1850, New York, Alfred A. Knopf, 2005.

Kandiyoti, Deniz, "Post-Colonialism Compared: Potentials and Limitations in the Middle East and Central Asia", International Journal of Middle East Studies, 35/2 (2002), p. 279-297.

Kavalski, Emilian, India and Central Asia: The Mythmaking and International Relations of a Rising Power, London, Tauris Academic Studies, 2010 (The Library of International Relations 47).

Kazimzade, E., "In the Margins: Minority Education in Central Asia», Khazar Journal of Humanities and Social Sciences, 14/1 (2011), p. 5-20.

Kemp, P. M., Bharat-Rus: The Story of 800 Years of Friendship, Delhi, ISCUS, 1958. 
Kemper, Michael, Conerman, Stephen (eds), The Heritage of Soviet Oriental Studies, London, Routledge, 2011 (Contemporary Russia and Eastern Europe Series 25).

Khalid, Adeeb, "Backwardness and the Quest for Civilization: Early Soviet Central Asia in Comparative Perspective", Slavic Review, 65/3 (2006), p. 231-251.

-, "Introduction: Locating the (Post-)colonial in Soviet History", Central Asian Survey, 26/4 (2007), p. 465-473.

—, "The Soviet Union as an Imperial Formation: A View from Central Asia", in Imperial Formations, ed. by A. Stoler, C. McGranahan, P. Perdue, Santa Fe, School of Advanced Research Press, 2007, p. 113-139.

-, "Central Asia between the Ottoman and the Soviet Worlds", Kritika: Explorations in Russian and Eurasian History, 12/2 (2011), p. 451-476.

Khodarkovsky, Michael, Russia's Steppe Frontier: The Making of a Colonial Empire, 1500-1800, Bloomington, Indiana University Press, 2002.

KIng, Richard, Orientalism and Religion: Postcolonial Theory, India and "the Mystic East", London/New York, Routledge, 1999.

Kopf, David, "Hermeneutics Versus History", The Journal of Asian Studies, 39/3 (1980), p. 495-506.

Korjukaeva, M. A., Russko-indijskie kul'turnye svjazi vtoroj poloviny $X I X$ - načala $X X v v$., thèse kandidatskaja en culturologie, SanktPeterburg, 2007.

Kotovsкij, G. G., Hejfetc, A. N., Šastitкo, P. M. (éds), SSSR i Indija, Moskva, Nauka, 1987.

Kritika: Models on the Margins: Russia and the Ottoman Empire, special issue of Kritika. Explorations in Russian and Eurasian History, 12/2 (2011).

Lally, Jagjeet, "Going Global: Recent Histories of Central Asia", Central Asian Survey, 31/1 (2012), p. 93-97.

Lardinois, Roland, L'invention de l'Inde. Entre ésotérisme et science, Paris, CNRS-Editions, 2007.

LARUelle, Marlène, Mythe aryen et rêve impérial dans la Russie tsariste, Paris, CNRS-Editions, 2005.

—, La quête d'une identité impériale: le néo-eurasisme dans la Russie contemporaine, Paris, Petra, 2007. 
—, Russian Eurasianism: An Ideology of Empire, Washington, Woodrow Wilson Press/Johns Hopkins University Press, 2008.

—, "Le paradigme du colonialisme en Asie centrale postsoviétique", L'homme et la société, 174 (2009), p. 27-40.

Laruelle, Marlène, Peyrouse, Sébastien, L'Asie centrale à l'aune de la mondialisation. Une approche géoéconomique, Paris, Armand Colin, 2010.

-, Mapping Central Asia: Indian Perceptions and Strategies, Farnham, Ashgate, 2011.

Laruelle, Marlène, Peyrouse, Sébastien, Huchet, Jean-François, Balci, Bayram, China and India in Central Asia: A New "Great Game"?, Basingstoke, Palgrave Macmillan, 2010.

Leach, Edmund, Muknerjee, S. N. (eds), Elites in South Asia, Cambridge, Cambridge University Press, 1970.

Lelyveld, David, "The Fate of Hindustani: Colonial Knowledge and the Project of a National Language", in Orientalism and the Postcolonial Predicament, ed. by C. Breckenridge, P. van der Veer, Philadelphia, University of Philadelphia Press, 1993, p. 189-214.

-, "Colonial Knowledge and the Fate of Hindustani", Comparative Studies in Society and History, 35/4 (1993), p. 665-682.

Levi, Scott C. (ed.), India and Central Asia: Commerce and Culture, 1500-1800, New Delhi, Oxford University Press, 2007 (Debates in Indian History and Society Series).

LÉVI, Sylvain, "Les parts respectives des nations occidentales dans les progrès de l'indianisme", in Mémorial Sylvain Lévi, éd. par L. Renou, Paris, Hartmann, 1937 [1924], p. 107-117.

Lieven, Dominic, Empire: The Russian Empire and its Rivals, London, John Murray, 2000.

Lopez, Donald S., Curators of the Buddha: The Study of Buddhism under Colonialism, Chicago, The University of Chicago Press, 1995.

Lorenzen, David N., "Who Invented Hinduism?", Comparative Studies in Society and History, 41/4 (1999), p. 630-659.

Lysenko, V., "Oktjabrina Fëdorovna Volkova. 23.01.1926-22.10.1988 ", in Smarlanam, Pamjati Oktjabriny Fëdorovny Volkovoj, éd. par V. G. Lysenko, Moskva, Naučnaja literatura, 2006, p. 12.

MacKenzie, John M., Orientalism: History, Theory and the Arts, Manchester, Manchester University Press, 1995. 
Martin, Terry, The Affirmative Action Empire: Nations and Nationalism in the Soviet Union, 1923-1939, Ithaca, Cornell University Press, 2001.

Matsuzato, Kimitaka, «Introduction», in Imperiology: From Empirical Knowledge to Discussing the Russian Empire, Sapporo, Slavic Research Center, 2007, p. 5-16.

Meaux, Lorraine de, La Russie et la tentation de l'Orient, Paris, Fayard, 2010.

Michaels, Paula, Curative Powers: Medicine and Empire in Stalin's Central Asia, Pittsburgh, Pittsburgh University Press, 2003.

Miller, Aleksej (éd.), Rossijskaja imperija v sravnitel'noj perspektive: sbornik statej, Moskva, Novoe izdatel'stvo, 2004.

Miller, Aleksei, Rieber, Alfred J. (eds), Imperial Rule, Budapest, Central European University Press, 2004.

Millward, J., Eurasian Crossroads: A History of Xinjiang, New York, Columbia University Press, 2007.

Mittal, M., In the Soul's Twilight: Remembering Tyutchev, New Delhi, 2005.

Mizuno, Hiromi, Science for the Empire: Scientific Nationalism in Modern Japan, Stanford, Stanford University Press, 2009.

Morrison, Alexander S., Russian Rule in Samarkand, 1868-1910: A Comparison with British India, New York, Oxford University Press, 2008.

—, "Applied Orientalism” in British India and Tsarist Turkestan", Comparative Studies in Society and History, 51/3 (2009), p. 619-647.

Motadel, David, "Islam and the European Empires", The Historical Journal, 55/3 (2012), p. 831-856.

Mrathuzina, G. F., Rossijsko-indijskie otnošenija v otečestvennoj istoriografii, thèse kandidatskaja en histoire, Kazan', 2001.

MüHlfried, Florian, Sokolovskiy, Sergey (eds), Exploring the Edge of Empire: Soviet Era Anthropology in the Caucasus and Central Asia, Zürich, Lit Verlag, 2011 (Halle Studies in the Anthropology of Eurasia 25).

Mukerji, Abhijit, "European Jones and Asiatic Pandits", Journal of the Asiatic Society, 27/1 (1985), p. 43-58.

Munhopadhyay, Amartya, India in Russian Orientalism: Travel Narratives and Beyond, New Delhi, Manohar, 2013. 
Nandy, Ashis, The Intimate Enemy: Loss and Recovery of Self Under Colonialism, Delhi, Oxford University Press, 1983.

-, Creating a Nationality: The Ramjanmabhumi Movement and Fear of the Self, New Delhi, Oxford University Press, 2003.

NeHru, Jawaharlal, Soviet Russia: Some Random Sketches and Impressions, Allahabad, Allahabad Law Journal Press, 1928.

—, The Discovery of India, New Delhi, Oxford University Press, 1982.

Newby, L., The Empire and the Khanate: A Political History of Qing Relations with Khoqand c. 1760-1860, Leiden, Brill, 2005.

Northrop, Douglas, Veiled Empire: Gender and Power in Stalinist Central Asia, Ithaca, 2004.

Olofsson, Kerstin, From Orientalism to Postcoloniality, Research Reports: 1, Södertörns högskola (University college), 2008.

Palumbo, Patrizia (ed.), A Place in the Sun: Africa in Italian Colonial Culture from Post-Unification to the Present, Berkeley, University of California Press, 2003.

Pandey, Gyanendra, Remembering Partition: Violence, Nationalism and History in India, Cambridge, Cambridge University Press, 2001.

PARK, Robert E., "Human Migration and the Marginal Man", American Journal of Sociology, 33/6 (1928), p. 881-893.

Peabody, Norbert, Hindu Kingship and Polity in Precolonial India, Cambridge, Cambridge University Press, 2003.

Petrova, E. Ju., Rossijsko-indijskii istoričeskie svjazi v dokumentah $i$ materialah arhivov L. N. Tolstogo iz muzeja-usad'by "Jasnaja Poljana", thèse kandidatskaja en histoire, Moskva, 2003.

Pianciola, N., Stalinismo di frontiera: colonizzazione agricola, sterminio dei nomadi e costruzione statale in Asia centrale, 1905-1936, Roma, Viella, 2009.

Pinch, William, "Review: Same Difference in India and Europe», History and Theory, 38/3 (1999), p. 389-407.

Poe, Marshall, A People Born to Slavery: Russia in Early Modern Ethnography, 1478-1750, Ithaca, Cornell University Press, 2000.

Pollock, Sheldon, «Deep Orientalism? Notes on Sanskrit and Power beyond the Raj", in Orientalism and the Postcolonial Predicament, ed. by C. Breckenridge, P. van der Veer, Philadelphia, University of Philadelphia Press, 1993, p. 76-115.

- (ed.), German Orientalism, special issue of Comparative Studies of South Asia, Africa and the Middle East, 24/2 (2004). 
-, The Language of the Gods in the World of Men: Sanskrit, Culture and Power in Premodern India, Berkeley, University of California Press, 2006.

Pondopoulo, Anna, Les Orients dans la culture russe, Toulouse, Université de Toulouse, 2012 (Slavica Occitania 35).

Pouillon, François, Vatin, Jean-Claude (éds), Après l'orientalisme: l'Orient créé par l'Orient, Paris, IISMM : Karthala, 2011.

Prakash, Gyan, "Post-Orientalist Histories of the Third World: Perspectives from Indian Historiography", Comparative Studies in Society and History, 32/2 (1990), p. 383-408.

Rabault-Feuerhahn, Pascale, "Voyages d'études et migrations savantes: Paris, lieu fondateur et provisoire de l'indianisme allemand", Revue germanique internationale, 7 (2008), p. 139-156.

RaJ, Kapil, Relocating Modern Science: Circulation and the Construction of Knowledge in South Asia and Europe, 1650-1900, New York, Palgrave Macmillan, 2007.

Raychaudhuri, Thapan, Europe Reconsidered: Perceptions of the West in Nineteenth-Century Bengal, Delhi, Oxford University Press, 1988. Regamey, Constantin, "The Meaning and Significance of Spirituality in Europe and in India", Philosophy East and West, 10/3-4 (19601961), p. 105-133.

—, «Un pionnier vaudois des études indiennes: Antoine-Louis de Polier", in Mélanges offerts à Monsieur Georges Bonnard, professeur honoraire de l'Université de Lausanne, à l'occasion de son quatrevingtième anniversaire, Genève, Droz, 1966, p. 183-209.

—, "Hommage à Constantin Régamey", Etudes Asiatiques, 35/2 (1981), p. 5-17.

Remnev, A., "Kolonial'nost', postkolonial'nost' i “istoričeskaja politika” v sovremennom Kazahstane", Ab Imperio, 1 (2011), p. 169-205.

Reynolds, Michael A., Shattering Empires: The Clash and Collapse of the Ottoman and Russian Empires, 1908-1918, Cambridge, Cambridge University Press, 2011.

Rocher, Rosane, "The Career of Rādhākānta Tarkavāgīśa, an Eighteenth-Century Pandit in British Employ", Journal of the American Oriental Society, 109/4 (1989), p. 627-633.

—, "British Orientalism in the Eighteenth Century: The Dialectics of Knowledge and Government", in Orientalism and the 
Postcolonial Predicament, ed. by C. Breckenridge, P. van der Veer, Philadelphia, University of Philadelphia Press, 1993, p. 215-249.

Roerich, G. N., "Indology in Russia», The Journal of the Greater India Society, 12/2 (1945), p. 69-98.

Rousseva-Sokolova, Galina, "Behind and Beyond the Iron Curtain:

Reception of Hindi Literature in Eastern and Central Europe", in India in Translation through Hindi Literature, ed. by M. Burger, N. Pozza, Bern, Peter Lang, 2010, p. 235-246.

Sahadeo, Jeff, Russian Colonial Society in Tashkent, 1865-1923, Bloomington, Indiana University Press, 2007.

Sahni, Kalpana, Crucifying the Orient, Bangkok, White Orchid Press, 1997.

SAID, Edward, Orientalism: Western Conceptions of the East, New York, Routledge, 1978.

—, Culture and Imperialism, New York, Knopf, 1993.

Samaddara, Ranabira, The Marginal Nation: Transborder Migration from Bangladesh to West Bengal, New Delhi/Thousand Oaks, Sage Publications, 1999.

SARtori, Paolo, "Introduction: Dealing with States of Property in Modern and Colonial Central Asia", Central Asian Survey, 29/1 (2010), p. 1-8.

Savelli, Dany, Présence du bouddhisme en Russie, Slavica Occitania, 21 (2005).

—, «Des théosophes sur la route de Lhassa: Les carnets de voyage au Tibet de trois membres de l'expédition Roerich (1927-1928)», in Pèlerinages en Eurasie et au-delà, éd. par Kathy Rousselet, Slavica Occitania, 36 (2013), p. 127-158.

Schimmelpenninck van der Oye, David, Russian Orientalism: Asia in the Russian Mind from Peter the Great to the Emigration, New Haven/London, Yale University Press, 2010.

Schwab, Raymond, La renaissance orientale, Paris, Payot, 1950.

Serebriany, Sergei, «Le roman en Russie et en Inde: Deux cas de transfert d'un objet culturel occidental dans une culture non occidentale", Revue Germanique Internationale, 21 (2004), p. 149-161.

—, "Comparative Literature and Post-colonial Studies: An Outsider's View from Post-Soviet Moscow", in Building Bridges between India and Russia: A Festschrift for Prof. J. P. Dimri, ed. by Ram Das Akella, Kolkata, Power Publishers, 2012, p. 63-82. 
Sharp, Jane Ashton, Russian Modernism between East and West: Natal'ia Goncharova and the Moscow Avant-Garde, Cambridge, Cambridge University Press, 2006.

Shree, Gitanjali, «Writing Is Translating Is Writing Is Translating Is», in India in Translation through Hindi Literature, ed. by M. Burger, N. Pozza, Bern, Peter Lang, 2010, p. 267-276.

Silova, Iveta (ed.), Globalization on the Margins: Education and Postsocialist Transformations in Central Asia, Charlotte, Information Age Publishing, 2011.

Sivasundaram, Sujit, Nature and the Godly Empire: Science and Evangelical Mission, 1795-1850, Cambridge/New York, Cambridge University Press, 2005.

—, "A Christian Benares": Orientalism and Science in the Serampore Mission of Bengal», Indian Economic and Social History Review, 44 (2007), p. 111-145.

Smith, David, "Orientalism and Hinduism", in The Blackwell Companion to Hinduism, ed. by G. Flood, Malden, Blackwell, 2005, p. 45-63.

Smith, Stefan Halikowski (ed.), Reinterpreting Indian Ocean Worlds: Essays in Honour of Kirti N. Chaudhuri, Newcastle upon Tyne, Cambridge Scholars, 2011.

Spechler, Martin, "Central Asia on the Edge of Globalization", Challenge, 47/4 (2004), p. 62-77.

SpIvak, Gayatri Chakravorty, "Can the Subaltern Speak? Speculations on Widow-Sacrifice", in Marxism and the Interpretation of Culture, ed. by Cary Nelson, Larry Grossberg, Urbana, University of Illinois Press, 1988, p. 271-313.

-, "The Burden of English", in Orientalism and the Postcolonial Predicament, ed. by C. Breckenridge, P. van der Veer, Philadelphia, University of Philadelphia Press, 1993, p. 134-157.

Stacy, R. H., India in Russian Literature, Delhi, Varanasi, Patna and Madras, 1985.

Stasulane, Anita, Theosophy and Culture: Nicolas Roerich, Roma, Pontificia Università Gregoriana, 2005.

Stoler, Ann Laura, "Considerations on Imperial Comparisons", in Empire Speaks Out: Languages of Rationalization and SelfDescription in the Russian Empire, ed. by I. Gerasimov, J. Kusber, A. Semyonov, Leiden, Brill, 2009, p. 33-46. 
Stolte, Carolien, Fischer-Tiné, Harald, «Imagining Asia in India: Nationalism and Internationalism (ca. 1905-1940)», Comparative Studies in Society and History, 54/1 (2012), p. 65-92.

Sub Altera Specie, "Roundtable "Sub Altera Specie": A View at Postcolonial Paradigm from Inside Russian/Soviet History [Adrienne Edgar, Sonja Luehrmann, Sergej Abašin, Elena Gapova]", Ab Imperio, 2 (2008), p. 80-92.

Subrahmanyam, Sanjay, "An Eastern El-Dorado: The TirumalaTirupati Temple-complex in Early European Views and Ambitions, 1540-1600", in Syllables of Sky: Studies in South Indian Civilization in Honour of Velchuru Narayana Rao, ed. by D. Shulman, Delhi, 1995 p. 338-390.

-, "The Career of Colonel Polier and Late Eighteenth-Century Orientalism ", Journal of the Royal Asiatic Society, 10 (2000), p. 43-60.

—, "Par-delà l'incommensurabilité: Pour une histoire connectée des empires aux temps modernes", Revue d'histoire moderne et contemporaine, 54/5 (2007), p. 34-53.

-, "Monsieur Picart and the Gentiles of India», in The First Global Vision of Religion: Bernard Picart's "Ceremonies and Customs of All Peoples of the World”, ed. by L. Hunt, M. Jacob, W. Mijnhardt, Los Angeles, Getty Research Institute, 2010, p. 197-214.

Sugirtharajah, Sharada, Imagining Hinduism: A Postcolonial Perspective, London, Routledge, 2003.

Sundaram, Susmita, The Land of Thought: India as Ideal and Image in Konstantin Bal'mont's, PhD thesis, Columbus, The Ohio State University, 2004.

Sunderland, Willard, "What Is Asia to Us? Scholarship on the Tsarist "East" since the 1990s", Kritika: Explorations in Russian and Eurasian History, 12/4 (2011), p. 817-833.

Sweetman, Will, "The Prehistory of Orientalism: Colonialism and the Textual Basis for Bartholomäus Ziegenbalg's Account of Hinduism ", New Zealand Journal of Asian Studies, 6/2 (2004), p. 12-38.

TAgore, Rabindranath, Letters from Russia, Calcutta, Viśva-bharati, 1960.

Teltscher, Kate, India inscribed: European and British Writing on India, 1600-1800, Delhi/Calcutta, Oxford University Press, 1997. 
Thompson, Ewa, Imperial Knowledge: Russian Literature and Colonialism, Westport, Greenwood Press, 2000.

Titus, P. (ed.), Marginality and Modernity: Ethnicity and Change in PostColonial Balochistan, Karachi, Oxford University Press, 1996.

Tolz, Vera, Russia's Own Orient: The Politics of Identity and Oriental Studies in the Late Imperial and Early Soviet Periods, Oxford, Oxford University Press, 2011.

Toro, Alfonso de, «Post-Coloniality and Post-Modernity: Jorge Luis Borges: The Periphery in the Centre, the Periphery as the Centre, the Centre of the Periphery", in Borders and Margins: PostColonialism and Post-Modernism, ed. by F. de Toro, A. de Toro, K. Quinn, Frankfurt am Main, Vervuert, 1995, p. 11-43.

Toro, Fernando de, "From Where to Speak? Post-Modern/PostColonial Positionalities", in Borders and Margins: PostColonialism and Post-Modernism, ed. by F. de Toro, A. de Toro, K. Quinn, Frankfurt am Main, Vervuert, 1995, p. 131-148.

Tranum, Sam, Life at the Edge of the Empire: Oral Histories of Soviet Kyrgyzstan, Bishkek, private publisher, 2009.

Trautmann, Thomas R., Aryans and British India, Berkeley, University of California Press, 1997.

—, "Does India Have History? Does History Have India?", Comparative Studies in Society and History, 54/1 (2012), p. 174-205.

Uyama, Tomohiko, Asiatic Russia: Imperial Power in Regional and International Contexts, London/New York, Routledge, 2011.

Vallorani, Nicoletta, Monegato, Emanuele (eds), Orientalism/ Orientalisms: Altre Modernità, Rivista di studi letterari e culturali, 8 (November 2012).

Van der Burg, Corstiaan J. G., "The Place of Sanskrit in Neo-Hindu Ideologies: From Religious Reform to National Awakening", in Ideology and Status of Sanskrit: Contributions to the Status of the Sanskrit Language, ed. by J. E. M. Houben, Leiden, Brill, 1996, p. 367-381.

van der Veer, Peter, "Hindu Nationalism and the Discourse of Modernity: The Vishva Hindu Parishad", in Accounting for Fundamentalisms, ed. by M. Marty, S. Appleby, Chicago, University of Chicago Press, 1994, p. 653-669. 
—, Religious Nationalism: Hindus and Muslims in India, Berkeley/Los Angeles, University of California Press, 1994.

Varisco, Daniel Martin, Reading Orientalism: Said and the Unsaid, Seattle/London, University of Washington, 2007.

Vasil'kov, Ja. V., Sorokina, M. Ju., Ljudi i sud'by: Biobibliografičeskij slovar' vostokovedov - žertv političeskogo terror v sovetskij period (1917-1991), Sankt-Peterburg, 2003; pour une version plus large et régulièrement mise à jour, voir: <http://memory.pvost.org/ pages/index 2. html>.

VIgasin, A. A., "Istoriografija istorii Drevnej Indii », in Istoriografija istorii drevnego Vostoka. Iran, Srednjaj Azija, Indija, Kitaj, ed. V. I. Kuziščin, Sankt-Peterburg, Izd. "Aletejja», 2002, p. 77-162.

Vigasin, A. A. et al. (éds), Russko-indijskie otnošenija v XIX v. Sbornik arhivnyh dokumentov i materialov, Moskva, Vostočnaja literatura, 1997.

—, Russko-indijskie otnošenija v 1900-1917 gg. Sbornik arhivnyh dokumentov i materialov, Moskva, Vostočnaja literatura, 1999.

Wageman, Patty, Kouteinikova, Inessa, Russia's Unknown Orient: Orientalist Painting 1850-1920, with contributions by Olga Atroshchenko, Irina Bagdamian, Vladimir Bulatov, Inessa Kouteinikova, David Schimmelpenninck van der Oye, Karina Solovyeva, Groningen/Rotterdam, Groninger Museum/Nai Publishers, 2010.

Weisberger, Adam, "Marginality and its Directions», Sociological Forum, 7/3 (1992), p. 425-446.

Wennberg, Franz, On the Edge: Concept of Progress in Bukhara during the Rule of the Later Manghits, Uppsala, Universitet, 2013 (Studia Iranica Upsaliensia 22).

Wоковск, Ursula, German Orientalism: The Study of the Middle East and Islam from 1800 to 1945, New York, Routledge, 2009 (Culture and Civilization in the Middle East).

Wolff, Larry, Inventing Eastern Europe: The Map of Civilization on the Mind of Enlightenment, Stanford, Stanford University, 1994.

Young, Louise, Japan's Total Empire: Manchuria and the Culture of Wartime Imperialism, Berkeley, University of California Press, 1998.

Zarcone, Thierry (éd.), Inde-Asie centrale: routes du commerce et des idées, Cahiers d'Asie centrale 1/2 (1996). 
Zarinebaf, Fariba, Bennet, John, Davis, Jack L. (eds), A Historical and Economic Geography of Ottoman Greece: The Southwestern Morea in the Eighteenth Century, Princeton, American School of Classical Studies at Athens, 2005.

Zelenev, E. I., Kasevič, V. B., Vvedenie v vostokovedenie, SanktPeterburg, Université de Saint-Pétersbourg, Faculté orientale, 2010. 